ALEA, Lat. Am. J. Probab. Math. Stat. 13, 1123-1149 (2016)

DOI: $10.30757 /$ ALEA.v13-40

\title{
Geodesic rays in the uniform infinite half-planar quadrangulation return to the boundary
}

\author{
Erich Baur, Grégory Miermont and Loïc Richier \\ ENS Lyon, UMPA UMR CNRS 5669, 46, allée d'Italie, 69364 Lyon Cedex 07, France. \\ E-mail address: \{ erich.baur, gregory.miermont, loic.richier \} @ens-lyon.fr
}

\begin{abstract}
We show that all geodesic rays in the uniform infinite half-planar quadrangulation (UIHPQ) intersect the boundary infinitely many times, answering thereby a recent question of Curien. However, the possible intersection points are sparsely distributed along the boundary. As an intermediate step, we show that geodesic rays in the UIHPQ are proper, a fact that was recently established in Caraceni and Curien (2015) by a reasoning different from ours. Finally, we argue that geodesic rays in the uniform infinite half-planar triangulation behave in a very similar manner, even in a strong quantitative sense.
\end{abstract}

\section{Introduction}

The uniform infinite half-planar quadrangulation UIHPQ provides a natural model of (discrete) random half-planar geometry. It arises as a local limit of finitesize quadrangulations with a boundary, when the number of quadrangles and the size of the boundary tend to infinity in a suitable way. We give more precise statements with references in the next section.

The full-plane equivalent of the UIHPQ is the so-called uniform infinite planar quadrangulation (UIPQ), which was introduced by Krikun (2005), after Angel and Schramm (2003)'s pioneering work on triangulations. It is proved in Curien et al. (2013) that geodesic rays (i.e., infinite one-ended geodesics) starting from the root in the UIPQ satisfy a confluence property towards infinity (and, as it is also shown, towards the root): Almost surely, there exists an infinite set of vertices such that every geodesic ray emanating from the origin passes through all the vertices of this

Received by the editors May 15, 2016; accepted November 12, 2016.

2010 Mathematics Subject Classification. 05C80, 60J80.

Key words and phrases. Uniform infinite half-planar quadrangulation, geodesic rays, boundary. Acknowledgment of support. The research of EB was supported by the Swiss National Science Foundation grant P300P2_161011, and performed within the framework of the LABEX MILYON (ANR-10-LABX-0070) of Université de Lyon, within the program "Investissements d'Avenir" (ANR-11-IDEX-0007) operated by the French National Research Agency (ANR). GM is a member of Institut Universitaire de France, and acknowledges support of the grant ANR-14-CE25-0014 (GRAAL) and of Fondation Simone et Cino Del Duca. 
set. In other words, geodesic rays in the UIPQ are essentially unique, in the sense that the Gromov boundary of the UIPQ contains only a single point.

In a recent work Caraceni and Curien (2015), Caraceni and Curien showed that the analog coalescence property of geodesics holds in the half-planar model UIHPQ: There is with probability one an infinite sequence of distinct vertices, which are all hit by every geodesic ray emanating from the root. Our main result of this paper shows that this property holds in the UIHPQ in a very strong sense.

Theorem 1.1. Almost surely, every geodesic ray in the UIHPQ hits the boundary infinitely many times. More specifically, almost surely there is an infinite sequence of distinct vertices all lying on the boundary of the UIHPQ, such that every geodesic ray passes through every point of this sequence except maybe for a finite number.

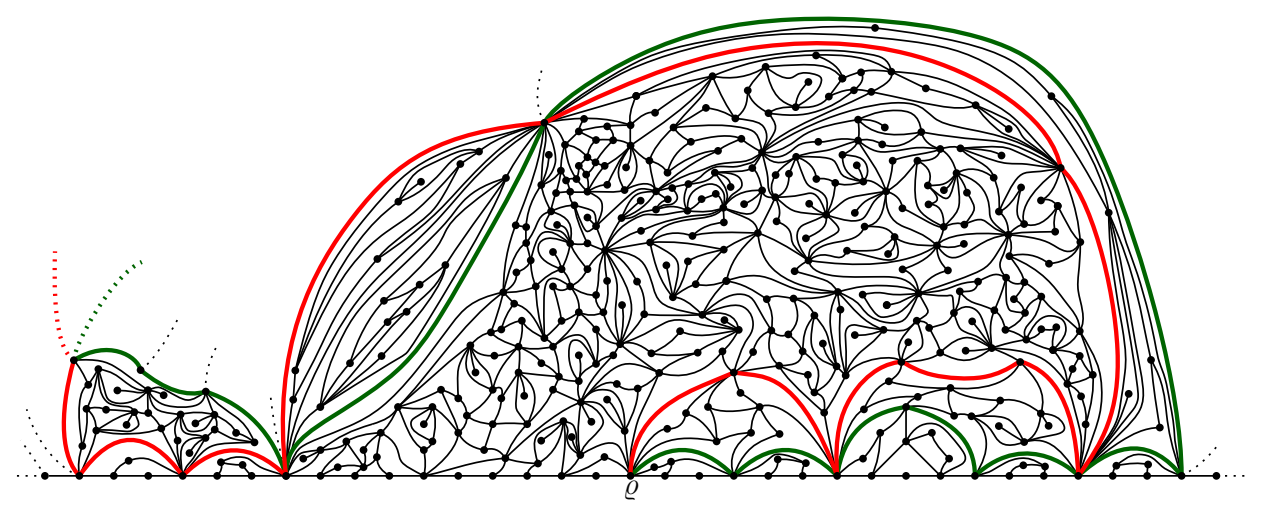

Figure 1.1. Artistic drawing of the UIHPQ (here, for simplicity, with a simple boundary) with two distinguished geodesics emanating from the root vertex $\rho$ called the maximal or leftmost geodesic (in red) and the minimal or rightmost geodesic (in green). All geodesic rays starting from $\rho$ lie in between the maximal and minimal geodesic. Their joint intersection points with the boundary are thus intersection points for any geodesic ray emanating from $\rho$.

After having introduced some notation, we will outline our strategy for proving Theorem 1.1 at the beginning of Section 3. In Section 4, we obtain more precise information on the set of times (and points) of intersection with the boundary, see Proposition 4.3. More specifically, by analyzing two distinguished geodesics starting from the root vertex, we will construct an infinite set of boundary vertices, which contains all possible points of intersection with any geodesic ray. See Figure 1.1. Our construction will imply that geodesic rays hit both "sides" of the boundary (see Section 2.2.3 for the exact terminology) infinitely many times; however, the time between two hits has a logarithmic tail. Section 5 contains an extension of our results to the uniform infinite half-planar triangulation UIHPT, see Theorem 5.3.

The UIHPQ considered here has a non-simple boundary, meaning that the boundary vertices cannot be connected by a simple curve. In other words, there are pinchpoints along the boundary. The analog of the UIHPQ with a simple boundary, which we denote by $\mathrm{UIHPQ}^{(s)}$ (see Angel and Curien, 2015; Curien and Miermont, 2015, 
and Angel, 2005 for the triangular analog), can be constructed by a pruning procedure applied to the UIHPQ, cf. Curien and Miermont (2015), and this construction will allow us to argue in Corollary 3.7 that our results on geodesics transfer to the $\mathrm{UIHPQ}^{(s)}$.

The uniform infinite planar quadrangulation UIPQ contains a distinguished infinite sequence of vertices, the so-called spine. This sequence can be interpreted as a self-avoiding infinite path in the UIPQ, which is, as it is shown in Curien et al. (2013), almost surely hit only a finite number of times by the collection of geodesic rays starting from the root. This result should be seen in comparison with our Theorem 1.1, see Remark 3.5 for more on this. In particular, in the UIPQ, there are self-avoiding paths of infinite length which are finally avoided by any geodesic ray. As our arguments leading to Theorem 1.1 show, such paths do not exist in the UIHPQ: Any infinite self-avoiding path in the UIHPQ must cross any geodesic ray infinitely often.

The fact that the spine is eventually left by the collection of geodesic rays emanating from the root is a key step in Curien et al. (2013) to prove the confluence property towards infinity, and our approach borrows to some extent from the ideas presented there.

We will rely on a Schaeffer-type encoding of the UIHPQ going back to Schaeffer (1998); Bouttier et al. (2004); Curien and Miermont (2015) in terms of uniformly labeled critical Galton-Watson trees, which are attached to the down-steps of a two-sided simple random walk. The key observation for Theorem 1.1 is expressed in Proposition 3.1. There, we find the exact distribution of the minimal label, which is attained in the trees attached to an excursion above -1 of the simple random walk. A related quantity is studied in Lemma 14 in Curien et al. (2013), see also Remark 3.5 below. In the last section, we argue that a variant of the Schaeffer-type encoding can be used to construct the uniform infinite half-planar triangulation UIHPT, and then a similar strategy works for the UIHPT as well, resulting in Theorem 5.3. In particular, somewhat surprisingly, we will see that geodesic rays in the UIHPT behave in a quantitatively very similar manner.

\section{The uniform infinite half-planar quadrangulation}

The UIHPQ is an infinite random quadrangulation with an infinite boundary, which comes equipped with an oriented root edge lying on the boundary. Let us first briefly recall the notion of planar quadrangulations with a boundary.

2.1. Planar maps and quadrangulations with a boundary. A finite planar map is a finite connected graph properly embedded in the two-dimensional sphere, that is, in such a way that edges intersect only at their endpoints. As usual, we regard two such maps as being equivalent, if they differ only by a homeomorphism that preserves the orientation of the sphere.

The faces of a planar map are the connected components of the complement of the union of its edges. The degree of a face is the number of its incident edges, where, as usual, an edge that lies entirely in a face is counted twice.

A planar map is a quadrangulation with a boundary, if all faces have degree four, except possibly one face called the root face, which can have an arbitrary (even) degree. The edges surrounding the root face form the boundary of the quadrangulation. We do not require the boundary to be a simple curve. 
The size of a quadrangulation with a boundary is the number (possibly infinite) of its non-root or inner faces. The size of the boundary, which is also called the perimeter of the map, is given by the degree of the root face. Note that since quadrangulations are bipartite, the perimeter is an even number.

Provided the perimeter is non-zero, in which case the map is seen as a single vertex map, we root such a quadrangulation by specifying one distinguished oriented edge on the boundary, in such a way that the root face lies to the right of that edge. The origin of the root edge is called the root vertex. We write $\mathcal{Q}_{f}$ for the set of all finite (rooted) quadrangulations with a boundary. Of course, if the perimeter of an element $q \in \mathcal{Q}_{f}$ is equal to four, we may view $q$ more naturally as a quadrangulation without boundary.

Equipped with the usual graph distance $d_{\mathrm{gr}}$, the vertex set $V(\mathfrak{m})$ of a rooted planar map $\mathfrak{m}$ is a pointed metric space. Let us next recall the so-called local topology on the set $\mathcal{Q}_{f}$ (or more generally, on the set of finite rooted maps).

Given a rooted planar map $\mathfrak{m}$ with root vertex $\varrho$, we denote by Ball ${ }_{r}(\mathfrak{m})$ for $r \geq 0$ the combinatorial ball of radius $r$, that is, the submap of $\mathfrak{m}$ containing all vertices $v$ of $\mathfrak{m}$ with $d_{\mathrm{gr}}(\varrho, v) \leq r$, together with the edges of $\mathfrak{m}$ connecting such vertices. Now if $\mathfrak{m}$ and $\mathfrak{m}^{\prime}$ are two rooted planar maps, the local distance between $\mathfrak{m}$ and $\mathfrak{m}^{\prime}$ is defined as

$$
d_{\text {map }}\left(\mathfrak{m}, \mathfrak{m}^{\prime}\right)=\left(1+\sup \left\{r \geq 0: \operatorname{Ball}_{r}(\mathfrak{m})=\operatorname{Ball}_{r}\left(\mathfrak{m}^{\prime}\right)\right\}\right)^{-1} .
$$

The local topology is the topology induced by $d_{\text {map }}$, and we write $\mathcal{Q}$ for the completion of $\mathcal{Q}_{f}$ with respect to $d_{\text {map }}$. Elements in $\mathcal{Q} \backslash \mathcal{Q}_{f}$ are called infinite quadrangulations with a boundary.

The UIHPQ $Q_{\infty}^{\infty}$ is a random (rooted) infinite quadrangulation with an infinite boundary, which can be obtained as a local limit of random elements in $\mathcal{Q}_{f}$, in the following ways.

Firstly, let $Q_{n}^{\sigma}$ be uniformly chosen among all rooted quadrangulations of size $n$ with a boundary of size $2 \sigma, \sigma \in \mathbb{N}=\{1,2, \ldots\}$. Curien and Miermont proved in Curien and Miermont (2015) that with respect to $d_{\text {map }}$,

$$
Q_{n}^{\sigma} \underset{n \rightarrow \infty}{\stackrel{(d)}{\longrightarrow}} Q_{\infty}^{\sigma}, \quad Q_{\infty}^{\sigma} \underset{\sigma \rightarrow \infty}{\stackrel{(d)}{\longrightarrow}} Q_{\infty}^{\infty} .
$$

Here, $Q_{\infty}^{\sigma}$ is the so-called uniform infinite planar quadrangulation with a boundary of length $2 \sigma$, see Curien and Miermont (2015) for a precise description. Similar convergences hold if $Q_{n}^{\sigma}$ is chosen uniformly among all rooted quadrangulations of size $n$ with a simple boundary of size $2 \sigma$, that is, if $Q_{n}^{\sigma}$ is a uniform rooted quadrangulation of the $2 \sigma$-gon with $n$ inner faces. In this case, the limiting map when first $n \rightarrow \infty$ and then $\sigma \rightarrow \infty$ is the uniform infinite planar quadrangulation with a simple boundary UIHPQ ${ }^{(s)}$, as alluded to above (see Angel and Curien, 2015 for details).

Secondly, the UIHPQ $Q_{\infty}^{\infty}$ arises also as the local limit of random elements in $\mathcal{Q}_{f}$ when the boundary grows simultaneously with the size of the map. More specifically, assume that $\sigma_{n}$ grows much slower than $n$. Then it is shown in Baur et al. (2016) that

$$
Q_{n}^{\sigma_{n}} \underset{n \rightarrow \infty}{\stackrel{(d)}{\longrightarrow}} Q_{\infty}^{\infty}
$$


In Curien and Miermont (2015), the UIHPQ $Q_{\infty}^{\infty}$ is constructed from an extended Schaeffer-type mapping applied to a so-called uniform infinite treed bridge of infinite length, and we will recall and work with this construction in the following section.

A new construction of the UIHPQ which is better suited to study the metric balls around the root has recently been given in Caraceni and Curien (2015). Although we will work with the first construction, we adopt some notation from there.

In the following section, we introduce certain deterministic objects which encode (non-random) infinite quadrangulations via a Schaeffer-type mapping. Randomized versions of these objects will then encode the UIHPQ.

\subsection{A Schaeffer-type construction.}

2.2.1. Well-labeled trees and infinite treed bridges. Recall the definition of a (rooted) finite planar tree $\tau$, see, e.g., Le Gall and Miermont (2012). We denote by $|\tau|$ the number of its edges and write $V(\tau)$ for the vertex set of $\tau$.

A well-labeled tree $(\tau, \ell)$ is a pair of a rooted planar tree $\tau$ and integer labels $\ell=(\ell(u))_{u \in V(\tau)}$, which are attached to the vertices of $\tau$, according to the following rule: Whenever $u, v \in V(\tau)$ are connected by an edge, then $|\ell(u)-\ell(v)| \leq 1$.

For $k \in \mathbb{Z}$, we let $\mathrm{LT}_{k}$ be the set of all finite well-labeled plane trees, whose root is labeled $k$. The set of all well-labeled plane trees is denoted $\mathrm{LT}=\cup_{k \in \mathbb{Z}} \mathrm{LT}_{k}$.

As in Curien and Miermont (2015) or Caraceni and Curien (2015), we will work with so-called treed bridges. We will only need their infinite versions, which we define next. First, an infinite bridge is a two-sided sequence $\mathrm{b}=(\mathrm{b}(i): i \in \mathbb{Z})$ with $\mathrm{b}(0)=0$ and $|\mathrm{b}(i+1)-\mathrm{b}(i)|=1$. An index $i$ for which $\mathrm{b}(i+1)=\mathrm{b}(i)-1$ is called a down-step of $\mathrm{b}$. The set of all down-steps of $\mathrm{b}$ is denoted DS(b).

Definition 2.1. We call infinite treed bridge a pair (b,T), where $\mathrm{b}$ is an infinite bridge and $T$ is a mapping from DS(b) to LT with the property that $T(i) \in \mathrm{LT}_{\mathrm{b}(i)}$, i.e., $T(i)$ is a well-labeled tree whose root has label $\mathrm{b}(i)$.

We write $\mathrm{TB}^{-\infty}$ for the set of all infinite treed bridges which have the property that $\inf _{i \in \mathbb{Z}_{+}} \mathrm{b}(i)=-\infty$ and $\inf _{i \in \mathbb{Z}_{-}} \mathrm{b}(i)=-\infty$, where $\mathbb{Z}_{+}=\{0,1,2, \ldots\}, \mathbb{Z}_{-}=$ $\{\ldots,-2,-1,0\}$.

2.2.2. The Bouttier-Di Francesco-Guitter mapping. We now construct a mapping $\Phi$, which we call the Bouttier-Di Francesco-Guitter mapping, that sends elements in $\mathrm{TB}^{-\infty}$ to infinite quadrangulations with an infinite boundary. The uniform infinite half-planar quadrangulation UIHPQ is then obtained from applying $\Phi$ to a random element $\left(\mathrm{b}_{\infty}, T_{\infty}\right)$ in $\mathrm{TB}^{-\infty}$, whose law we specify in the next section.

We stress that usually (e.g., in Curien and Miermont, 2015, or in Caraceni and Curien, 2015), the Bouttier-Di Francesco-Guitter mapping is first introduced as a bijection between finite versions of treed bridges and (rooted and pointed) finitesize quadrangulations with a boundary. Then it is argued that the mapping can be extended to elements in $\mathrm{TB}^{-\infty}$, yielding infinite quadrangulations. However, since we will here only work with infinite quadrangulations, we directly describe the mapping as a function

$$
\Phi: \mathrm{TB}^{-\infty} \longrightarrow \mathcal{Q} \text {. }
$$

Let $(\mathrm{b}, T) \in \mathrm{TB}^{-\infty}$. It is convenient to work with the following representation of $(\mathrm{b}, T)$ in the plane: We identify $\mathrm{b}=(\mathrm{b}(i): i \in \mathbb{Z})$ with the labeled bi-infinite line, which is obtained from connecting the neighboring vertices of $\mathbb{Z}$ by edges and 
assigning to $i \in \mathbb{Z}$ the label $\mathrm{b}(i)$. Then we graft a proper embedding of the tree $T(i)$ for $i \in \mathrm{DS}(\mathrm{b})$ to the vertex $i$ in the upper half-plane, by identifying the root of $T(i)$ with the vertex $i$. See Figure 2.2. Note our small abuse of notation: We denote here by $i \in \mathrm{DS}(\mathrm{b})$ an index of $\mathrm{b}$ as well as a vertex of the representation of b.

The vertex set of such a representation of $(b, T)$ is therefore given by $\mathbb{Z}$ and the union of the tree vertices of $T(i), i \in \mathrm{DS}(\mathrm{b})$, where we interpret the root of $T(i)$ and the vertex $i \in \mathbb{Z}$ as one and the same vertex. Following the wording of Caraceni and Curien (2015), we call the vertices which belong to the trees $T(i), i \in \mathrm{DS}(\mathrm{b})$, real vertices, and the vertices $j \in \mathbb{Z}$ above which no trees are grafted, i.e., the vertices $j$ that do not correspond to down-steps of $\mathrm{b}$, phantom vertices. A corner of (the representation of) (b, $T$ ) is an angular sector between two consecutive edges, in the clockwise contour or left-to-right order. Henceforth we shall consider only real corners, i.e., corners that are incident to real vertices and lie in the upper halfplane. By a small abuse of notation, given a vertex $v \in T(i), i \in \mathrm{DS}(\mathrm{b})$, we shall simply write $\ell(v)$ for its label, and we let $\ell(c)=\ell(v)$ if $c$ is a corner incident to $v$.

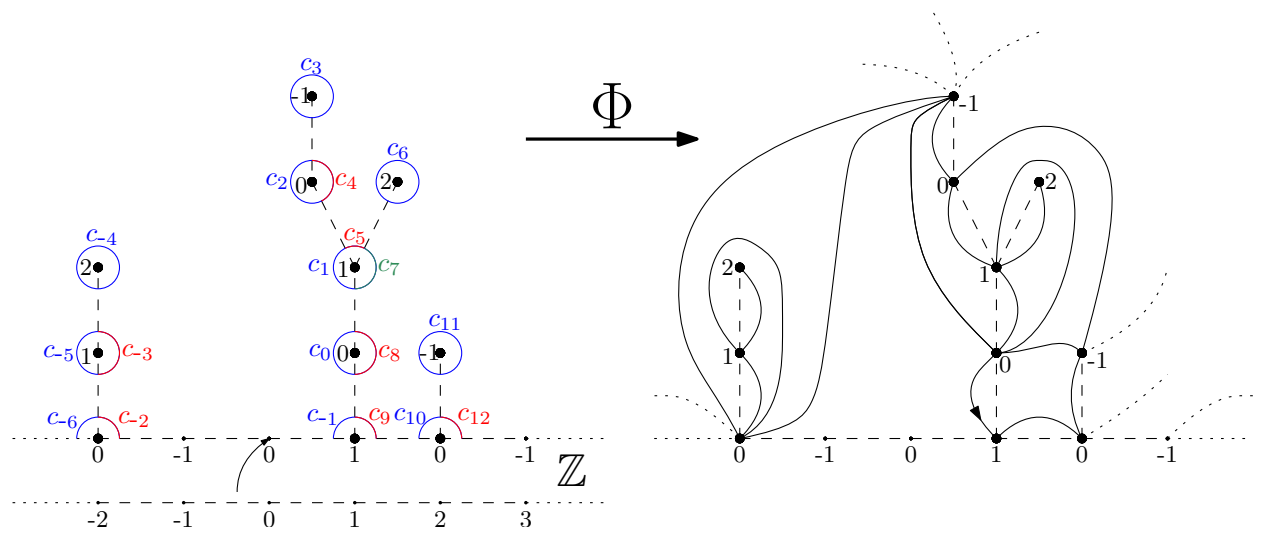

Figure 2.2. The Bouttier-Di Francesco-Guitter mapping. Vertex 0 of $\mathbb{Z}$ is indicated by an arrow. The visible trees are attached to the vertices $-2,1$ and 2 of $\mathbb{Z}$, which are labeled 0,1 and 0 , respectively. These vertices correspond to down-steps of the bridge.

We now consider the bi-infinite sequence of corners $\left(c_{i}\right)_{i \in \mathbb{Z}}$ obtained from ordering the real corners of (b, $T$ ) according to the left-to-right order, where we agree that $c_{0}$ is the left-most real corner with label 0 , which appears in $T(i), i \in \operatorname{DS}(\mathrm{b}) \cap \mathbb{Z}_{+}$. See again Figure 2.2. For $i \in \mathbb{Z}$, we denote by $\operatorname{succ}\left(c_{i}\right)$ the first corner among $c_{i+1}, c_{i+2}, \ldots$, which has label $\ell\left(c_{i}\right)-1$. Note that such a corner always exists, since $\inf _{i \in \mathbb{Z}_{+}} \mathrm{b}(i)=-\infty$. We call $\operatorname{succ}\left(c_{i}\right)$ the successor of $i$. As indicated on the right side of Figure 2.2, we draw for every $i \in \mathbb{Z}$ an arc between the corner $c_{i}$ and $\operatorname{succ}\left(c_{i}\right)$ in the upper half-plane, in such a way that arcs do only possibly intersect at their endpoints. We finally erase the phantom vertices and the edges that stem from the representation of $(\mathrm{b}, T)$. We obtain a locally finite quadrangulation $M$ with an infinite boundary $\partial M$, which we root in the (oriented) edge that corresponds to the first step of the bridge to the right of 0 . A detailed explanation of this correspondence is given in the next section. In other words, the root face that lies 
to the right of the root edge has infinite degree, and the edges surrounding it form the (infinite) boundary $\partial M$ of the map.

We let $\Phi((\mathrm{b}, T))=M$ be the rooted infinite quadrangulation with an infinite boundary obtained in this way.

2.2.3. Identification of the boundary. If we identify $\mathbb{Z}$ with the bi-infinite line by connecting neighboring vertices with an edge, then the Bouttier-Di FrancescoGuitter mapping establishes a one-to-one correspondence between the edges of $\mathbb{Z}$ and those of the boundary $\partial M$ of $M=\Phi((\mathrm{b}, T))$, as it is visible in Figures 2.2 and 2.3. More precisely, for a given (b, $T)$, we define a function

$$
\varphi: \mathbb{Z} \rightarrow V(\partial M)
$$

as follows: Vertex $i \in \mathbb{Z}$ of the representation of $\mathbf{b}$ (which is labeled $\mathbf{b}(i)$ ) is mapped to itself, if $i$ is a real vertex. By definition, this is the case if and only if $i \in \operatorname{DS}(b)$. Otherwise, we search for the next real corner to the right of $i$ which has label $\mathrm{b}(i)$, and define $\varphi(i)$ to be the vertex incident to it. Then the edge $\{i, i+1\}$ of $\mathbb{Z}$ corresponds to a unique edge from $\varphi(i)$ to $\varphi(i+1)$ of $\partial M$, and the assignment is one-to-one. Instead of being more formal, we refer to Figure 2.3.

We will call $\varphi\left(\mathbb{Z}_{-}\right)$and $\varphi\left(\mathbb{Z}_{+}\right)$the left and right part of the boundary of $M$, respectively. Of course, $\partial M=\varphi\left(\mathbb{Z}_{-}\right) \cup \varphi\left(\mathbb{Z}_{+}\right)$. Moreover, $M$ is rooted in the (oriented) edge between $\varphi(0)$ and $\varphi(1)$.

2.2.4. Construction of the UIHPQ. Recall the definition of $\mathbf{L T}_{k}$ for $k \in \mathbb{Z}$. Let $\rho_{k}$ be the Boltzmann measure on $\mathrm{LT}_{k}$ given by $\rho_{k}((\tau, \ell))=12^{-|\tau|} / 2$. The measure $\rho_{k}$ is the law of a so-called uniformly labeled critical geometric Galton-Watson tree. This means that if $(\tau, \ell)$ is distributed according to $\rho_{k}$, then $\tau$ has the law of a GaltonWatson tree with a geometric offspring distribution of parameter 1/2. Moreover, conditionally on $\tau, \ell: V(\tau) \rightarrow \mathbb{Z}$ is the random labeling of $\tau$ such that the root receives label $k$, and independently for each edge $e=\{u, v\}$ of $\tau, \ell(u)-\ell(v)$ is uniformly distributed over $\{-1,0,1\}$. We refer, e.g., to Le Gall and Miermont (2012, Section 2.2) for more details.

Let $\mathrm{b}_{\infty}=\left(\mathrm{b}_{\infty}(i): i \in \mathbb{Z}\right)$ be a two-sided simple symmetric random walk with $\mathrm{b}_{\infty}(0)=0$, that is, $\left(\mathrm{b}_{\infty}(i): i \in \mathbb{Z}_{+}\right)$and $\left(\mathrm{b}_{\infty}(i): i \in \mathbb{Z}_{-}\right)$are two independent simple symmetric random walks starting from 0 .

Conditionally on $\mathrm{b}_{\infty}$, define a (random) function $T_{\infty}: \mathrm{DS}\left(\mathrm{b}_{\infty}\right) \rightarrow$ LT by letting $T_{\infty}(i)$ for $i \in \mathrm{DS}\left(\mathrm{b}_{\infty}\right)$ be a well-labeled tree with law $\rho_{\mathrm{b}_{\infty}(i)}$, independently in $i \in \mathrm{DS}\left(\mathrm{b}_{\infty}\right)$.

We call the random element $\left(\mathrm{b}_{\infty}, T_{\infty}\right)$ of $\mathrm{TB}^{-\infty}$ a uniform infinite treed bridge.

Definition 2.2. The UIHPQ $Q_{\infty}^{\infty}=\left(V\left(Q_{\infty}^{\infty}\right), d_{\mathrm{gr}}, \varrho\right)$ is the random infinite quadrangulation with an infinite boundary obtained from applying the Bouttier-Di Francesco-Guitter mapping to a uniform infinite treed bridge $\left(\mathrm{b}_{\infty}, T_{\infty}\right)$, i.e.,

$$
Q_{\infty}^{\infty}=\Phi\left(\left(\mathrm{b}_{\infty}, T_{\infty}\right)\right)
$$

We will write $\ell_{\infty}(v)$ for the label of a vertex $v \in V\left(T_{\infty}(i)\right), i \in \mathrm{DS}\left(\mathrm{b}_{\infty}\right)$, which we also identify with a vertex of $Q_{\infty}^{\infty}$ via the Bouttier-Di Francesco-Guitter mapping. 
2.3. Geodesics in the UIHPQ. Let $G=(V(G), E(G))$ be a graph. A geodesic in $G$ is a path of possibly infinite length, which visits a sequence (or chain) of vertices $\gamma=$ $(\gamma(0), \gamma(1), \ldots)$ of $G$ such that for $i, j \in \mathbb{Z}_{0}$ for which $\gamma$ is defined, $d_{\mathrm{gr}}(\gamma(i), \gamma(j))=$ $|i-j|$. An infinite geodesic $\gamma$ with $\gamma(0)=v \in V(G)$ is called a geodesic ray started at $v$.

Note that we view a geodesic as a sequence of concatenated edges. In particular, if $G$ is a non-simple graph as in the case of the UIHPQ, a geodesic is usually not specified by its vertices alone.

Let $(\mathrm{b}, T) \in \mathrm{TB}^{-\infty}$ be an infinite treed bridge. We will now define particular geodesic rays in the infinite quadrangulation $\Phi((\mathrm{b}, T))$. Recall the definition of the sequence of corners $\left(c_{i}\right)_{i \in \mathbb{Z}}$ obtained from ordering the real corners of $(\mathrm{b}, T)$ according to the contour order, as well as the definition of the successor-mapping; see Section 2.2.2. We write $\operatorname{succ}^{(i)}$ for the $i$-fold composition of the successormapping and denote by $\mathcal{V}(c)$ the vertex incident to the corner $c$.

Definition 2.3 (Maximal geodesic). Let (b, $T) \in \mathrm{TB}^{-\infty}$, and let $v \in V(\Phi((\mathrm{b}, T))$ ) be a vertex of the quadrangulation associated to (b, $T$ ). Let $c$ be the leftmost (real) corner of $(\mathrm{b}, T)$ incident to $v$. Then the maximal geodesic started at $v$ is given by the chain of vertices incident to the iterated successors of $c$, that is, $\gamma_{\max }^{v}(0)=v$, and then for $i \in \mathbb{N}$,

$$
\gamma_{\max }^{v}(i)=\mathcal{V}\left(\operatorname{succ}^{(i)}(c)\right)
$$

and with edges connecting $\operatorname{succ}^{(i)}(c)$ to $\operatorname{succ}^{(i+1)}(c)$ for $i \in \mathbb{Z}_{+}$.

We will simply write $\gamma_{\max }$ for the maximal geodesic started from the root $\varrho$. See Figure 2.3 for an illustration of the maximal geodesic in the UIHPQ. It is a direct consequence of the definition that maximal geodesics finally coalesce. Indeed, consider the first vertex incident to a corner $c_{i}$ for $i \in \mathbb{Z}_{+}$, which is visited by $\gamma_{\max }^{v}$. Let $v^{\prime}$ be the first vertex incident to a corner $c_{j}, j \geq i$, which is visited by $\gamma_{\max }$. Then $v^{\prime}$ is also visited by $\gamma_{\max }^{v}$, and from that moment on, $\gamma_{\max }^{v}$ and $\gamma_{\max }$ coincide.

Of special interest is the class of proper geodesics, which generalizes the construction of maximal geodesics, in the sense that the connecting edges do not necessarily emanate from leftmost corners.

Definition 2.4 (Proper geodesic). A geodesic ray $\gamma$ is proper, if for every $i \in \mathbb{Z}_{+}$,

$$
\ell(\gamma(i+1))=\ell(\gamma(i))-1
$$

It turns out that in the UIHPQ, almost surely every geodesic ray is proper. This fact has already been proved in Caraceni and Curien (2015), but we will give an alternative proof in Corollary 3.6. In particular, it makes sense to call maximal geodesics leftmost geodesics. In Section 4, we shall also consider minimal or rightmost geodesics.

\section{Proof of the main results}

To begin with, let us describe our general strategy for proving Theorem 1.1.

We will first show that the maximal geodesic $\gamma_{\max }$ hits both parts of the boundary of the UIHPQ infinitely many times, see Proposition 3.4 below. For that purpose, we will study the sets $\mathcal{R}_{+}$and $\mathcal{R}_{-}$of intersection times of $\gamma_{\max }$ with the right and left part of the boundary. It turns out that both $\mathcal{R}_{+}$and $\mathcal{R}_{-}$are regenerative sets. Moreover, we find a representation of these sets in terms of the infinite treed 


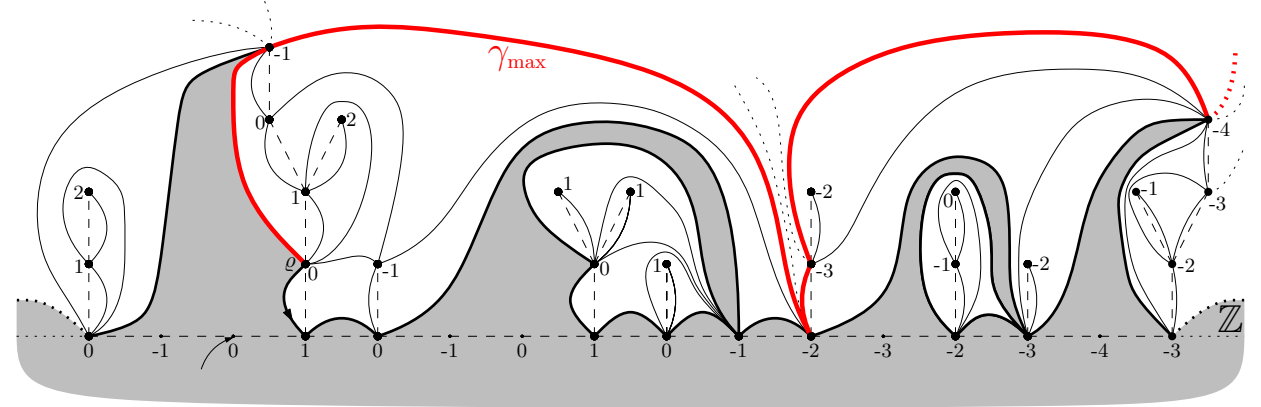

Figure 2.3. The UIHPQ and its maximal geodesic $\gamma_{\max }$.

bridge encoding the UIHPQ, which involves the minimal label attained in the trees between two subsequent minima of the bridge. The crucial step is formulated as Proposition 3.1 below, where we compute the exact distribution of such a minimal label. Once we know that $\gamma_{\max }$ touches both parts of the boundary infinitely often, we also know that every geodesic ray must cross $\gamma_{\max }$ infinitely many times. From this, we readily deduce that any geodesic ray is proper, as it was already shown in Caraceni and Curien (2015, Proposition 4.8) for geodesic rays started from the root vertex, by means different from ours. Since any proper geodesic ray lies finally in between $\gamma_{\max }$ and the boundary, an appeal to Proposition 3.4 allows us to conclude the proof of Theorem 1.1.

We first introduce some more notation. Let $(\mathrm{b}, T) \in \mathrm{TB}^{-\infty}$ be an infinite treed bridge. For $j \in \mathbb{Z}_{+}$, we write

$$
H_{j}(\mathrm{~b})=\inf \left\{m \in \mathbb{Z}_{+}: \mathrm{b}(m)=-j\right\}, \quad H_{j}^{\prime}=\sup \left\{m \in \mathbb{Z}_{-}: \mathrm{b}(m)=-j\right\}
$$

for the first time $\mathrm{b}$ hits $-j$ to the right of zero or to the left of zero, respectively. Note that both $H_{j}$ (b) and $H_{j}^{\prime}(\mathrm{b})$ are finite for each $j \in \mathbb{Z}_{+}$, almost surely.

Moreover, for $i \in \mathrm{DS}(\mathrm{b})$, we write $\ell_{i}=\left(\ell_{i}(u)\right)_{u \in V(T(i))}$ for the labels of the vertices of the tree $T(i) \in \mathrm{LT}_{\mathrm{b}(i)}$. Recall that if $r$ is the root vertex of $T(i)$, then $\ell_{i}(r)=\mathrm{b}(i)$.

For $j \in \mathbb{Z}_{+}$, we let

$$
\begin{aligned}
& \Delta_{j}((\mathrm{~b}, T))=\max _{i \in \mathrm{DS}(\mathrm{b}) \cap\left[H_{j}, H_{j+1}\right)}-\left(\min _{u \in V(T(i))} \ell_{i}(u)+j\right), \quad \text { and } \\
& \Delta_{j}^{\prime}((\mathrm{b}, T))=\max _{i \in \mathrm{DS}(\mathrm{b}) \cap\left[H_{j+1}^{\prime}, H_{j}^{\prime}\right)}-\left(\min _{u \in V(T(i))} \ell_{i}(u)+j\right),
\end{aligned}
$$

where $H_{j}=H_{j}\left(\mathrm{~b}_{\infty}\right)$, and $H_{j}^{\prime}=H_{j}^{\prime}\left(\mathrm{b}_{\infty}\right)$. In words, $\Delta_{j}((\mathrm{~b}, T)) \in \mathbb{Z}_{+}$is the absolute value of the minimal label shifted by $\left|\mathrm{b}\left(H_{j}\right)\right|=j$ in the trees $T(i)$ that are attached to the infinite bridge b on $\left[H_{j}, H_{j+1}\right)$. A similar interpretation holds for $\Delta_{j}^{\prime}((\mathrm{b}, T))$. We simply write $\Delta_{j}$ and $\Delta_{j}^{\prime}$ for the random numbers $\Delta_{j}\left(\left(\mathrm{~b}_{\infty}, T_{\infty}\right)\right)$ and $\Delta_{j}^{\prime}\left(\left(\mathrm{b}_{\infty}, T_{\infty}\right)\right)$, where $\left(\mathrm{b}_{\infty}, T_{\infty}\right)$ is a uniform infinite treed bridge as specified in Section 2.2.4. The strong Markov property shows that $\Delta_{j}$ has the same law as $\Delta_{0}$, and $\Delta_{j}^{\prime}$ has the same law as $\Delta_{0}^{\prime}$, for each $j \in \mathbb{Z}_{+}$. As we show next, their distributions can be computed explicitly. 
Proposition 3.1. We have for $m \in \mathbb{N}$,

$$
\mathbb{P}\left(\Delta_{0} \geq m\right)=\frac{1}{m+1}, \quad \text { and } \quad \mathbb{P}\left(\Delta_{0}^{\prime} \geq m\right)=\frac{1}{m+3} .
$$

Proof: We first consider $\Delta_{0}$. The statement for $\Delta_{0}^{\prime}$ will then follow from a symmetry argument. Let $m \in \mathbb{N}$. We set $g(m)=\mathbb{P}\left(\Delta_{0}<m\right)$. Moreover, let $h(m)=\mathbb{P}\left(\min _{u \in V(\tau)} \ell(u)>-m\right)$, where $(\tau, \ell)$ is distributed according to $\rho_{0}$; see Section 2.2.4. We decompose the path of $\mathrm{b}$ on $\left[0, H_{1}\right)$ into its excursions above 1 , as shown in Figure 3.4. For $\Delta_{0}$ to be smaller than $m$, the labels in every excursion above 1 have to be larger than $-(m+1)$, while the minimal label of the tree grafted to the last step of the excursion has to be larger than $-m$.

A standard application of the strong Markov property shows that these excursions, shifted by -1 , have the same law as b on $\left[0, H_{1}\right)$, so that the quantity $g(m)$ satisfies the recursive equation

$$
g(m)=\frac{1}{2} h(m) \sum_{k=0}^{\infty}\left(\frac{1}{2} g(m+1)\right)^{k}=\frac{h(m)}{2-g(m+1)} .
$$

We stress that (3.1) is in spirit of the arch decomposition as described in Section V.4.1 of Flajolet and Sedgewick (2009); see also (2.1) and (2.2) of Bouttier and Guitter (2012) for related decompositions.

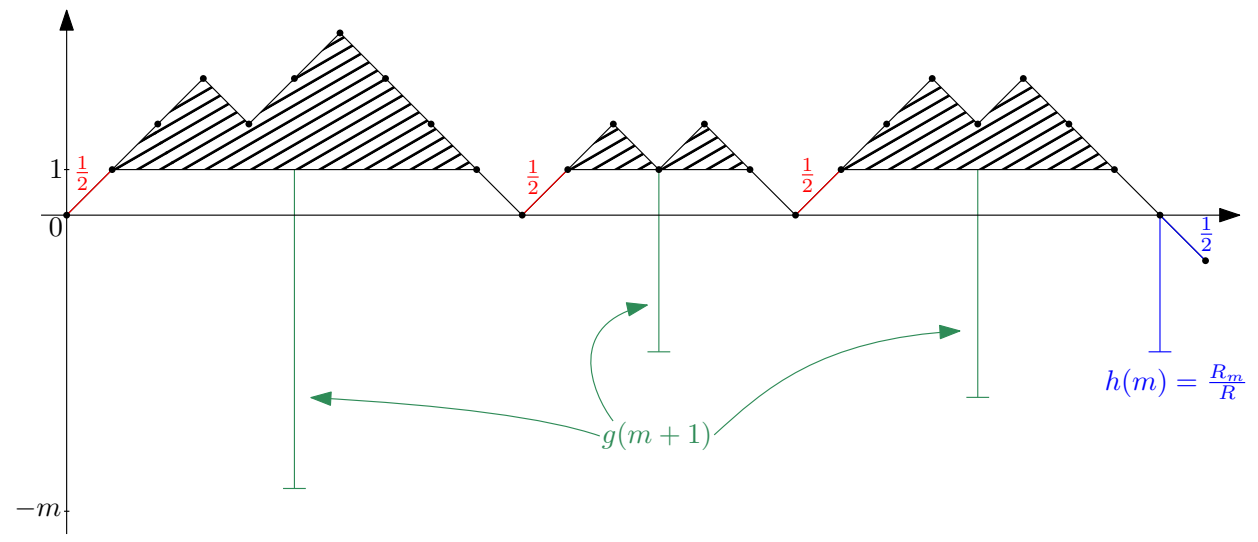

Figure 3.4. The decomposition of the probability $g(m)$.

From the Bouttier-Di Francesco-Guitter bijection for quadrangulations of a finite size, see, e.g., Bouttier et al. (2004), well-labeled trees are in bijection with rooted and pointed quadrangulations, the pointed vertex being at distance $\min _{u \in V(\tau)} \ell(u)-$ 1 from the root. In Bouttier and Guitter (2012), the generating function for quadrangulations with weight $g_{4}$ per face and distance less than or equal to $m$ between the root and the pointed vertex, called the distance-dependent two-point function and denoted $R_{m}$, is proved to satisfy (see Bouttier and Guitter, 2012, (6.18))

$$
R_{m}=R \frac{\left(1-y^{m}\right)\left(1-y^{m+3}\right)}{\left(1-y^{m+1}\right)\left(1-y^{m+2}\right)},
$$

where $R=R\left(g_{4}\right)=\lim _{m \rightarrow \infty} R_{m}$ is the generating function of rooted and pointed quadrangulations with weight $g_{4}$ per face, and $y=y\left(g_{4}\right)$ is the solution of the 
so-called characteristic equation (see Bouttier and Guitter, 2012, (6.17)). In our special case corresponding to a critical weight per face given by $g_{4, \mathrm{cr}}=1 / 12$, the solution of the characteristic equation simplifies to $y=1$. Taking the limit $y \uparrow 1$ in the last display, this implies

$$
R_{m}=R \frac{m(m+3)}{(m+1)(m+2)} .
$$

Since the partition function is given by $R$, we therefore get

$$
h(m)=\mathbb{P}\left(-\min _{u \in V(\tau)} \ell(u) \leq m-1\right)=\frac{R_{m}}{R}=1-\frac{2}{(m+1)(m+2)} .
$$

By the way, we note that $h(m)$ as already been calculated before in Chassaing and Durhuus (2006, Proposition 2.4); see Remark 3.3 below. Letting $f(m)=\mathbb{P}\left(\Delta_{0} \geq\right.$ $m)=1-g(m)$, we obtain from (3.1) and the last display

$$
f(m)-f(m+1)+f(m) f(m+1)=\frac{2}{(m+1)(m+2)} \quad \text { for all } m \in \mathbb{N} .
$$

Our claim about $\Delta_{0}$ now follows from the following

Lemma 3.2. Consider the non-linear system

$$
\left\{\begin{aligned}
f(m)-f(m+1)+f(m) f(m+1) & =\frac{2}{(m+1)(m+2)} \quad \text { for all } m \in \mathbb{N}, \\
f(0) & =1, \\
\lim _{m \rightarrow \infty} f(m) & =0 .
\end{aligned}\right.
$$

Then the only solution $f$ of $(3.3)$ with $f(m) \in(0,1)$ for all $m \in \mathbb{N}$ is given by $f(m)=1 /(m+1), m \in \mathbb{Z}_{+}$.

Proof: It is elementary to check that $f(m)=1 /(m+1), m \in \mathbb{Z}_{+}$, is a solution of (3.3) with $f(\mathbb{N}) \subset(0,1)$, so it remains to show uniqueness. We first prove the following statement:

If $f_{1}, f_{2}: \mathbb{Z}_{+} \rightarrow(0,1)$ are two solutions of (3.3) such that $f_{1}(m)<f_{2}(m)$ for some $m \in \mathbb{N}$, then $f_{1}(m+k)<f_{2}(m+k)$ for all $k \in \mathbb{Z}_{+}$.

Indeed, assume $f_{1}(m)<f_{2}(m)$ for some $m \in \mathbb{N}$. We show that then also $f_{1}(m+1)<$ $f_{2}(m+1)$. Since $f_{1}$ is a solution of (3.3), we can use (3.3) to express $f_{1}(m+1)$ in terms of $f_{1}(m)$ and obtain

$f_{1}(m+1)=\frac{(m+1)(m+2) f_{1}(m)-2}{(m+1)(m+2)\left(1-f_{1}(m)\right)}<\frac{(m+1)(m+2) f_{2}(m)-2}{(m+1)(m+2)\left(1-f_{2}(m)\right)}=f_{2}(m+1)$.

An iteration of the argument shows $f_{1}(m+k)<f_{2}(m+k)$ for all $k \in \mathbb{Z}_{+}$and hence (3.4).

Now assume there are two solutions $f_{1}, f_{2}: \mathbb{Z}_{+} \rightarrow(0,1)$ of $(3.3)$ with $f_{1} \neq f_{2}$. Then there exists $\varepsilon>0$ and $m \in \mathbb{N}$ such that $f_{2}(m)-f_{1}(m)>\varepsilon$ or $f_{1}(m)-f_{2}(m)>$ $\varepsilon$. By symmetry, we may assume the former. Since both $f_{1}$ and $f_{2}$ solve (3.3), we obtain for their difference

$f_{2}(m)-f_{1}(m)-\left(f_{2}(m+1)-f_{1}(m+1)\right)+f_{2}(m) f_{2}(m+1)-f_{1}(m) f_{1}(m+1)=0$.

By assumption, $f_{2}(m)-f_{1}(m)>\varepsilon$, which implies by (3.4) that

$$
f_{2}(m) f_{2}(m+1)-f_{1}(m) f_{1}(m+1)>0 .
$$

Therefore, we obtain from (3.5) that also $f_{2}(m+1)-f_{1}(m+1)>\varepsilon$. Iterating the argument, we see $\lim _{m \rightarrow \infty} f_{2}(m) \geq \varepsilon$, a contradiction to $\lim _{m \rightarrow \infty} f_{2}(m)=0$. 


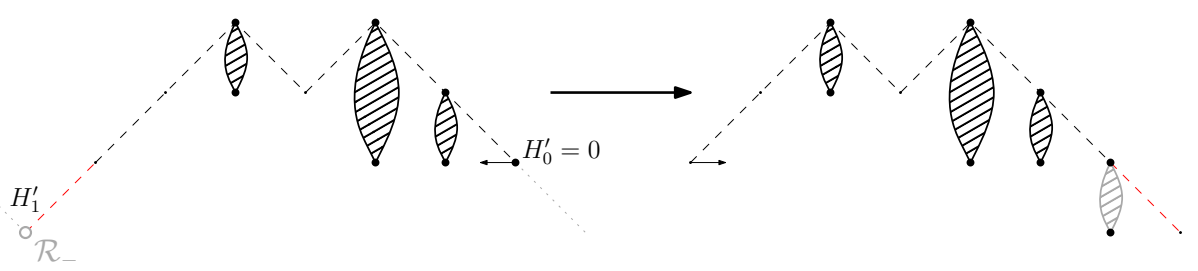

FiguRE 3.5. The symmetry argument between excursions of $b_{\infty}$.

We continue the proof of Proposition 3.1 and turn to the distribution of $\Delta_{0}^{\prime}$. By time-reversal, $\left(\mathrm{b}_{\infty}(i): H_{1}^{\prime}<i \leq 0\right)$ has the same law as $\left(\mathrm{b}_{\infty}(i): 0 \leq i<H_{1}\right)$. Moreover, down-steps $i$ of $\left(\mathrm{b}_{\infty}(i): H_{1}^{\prime}<i \leq 0\right)$ belong to $\mathrm{DS}\left(\mathrm{b}_{\infty}\right)$, and as shown in Figure 3.5, independent trees with law $\rho_{\mathrm{b}_{\infty}(i)}$ are assigned to them. However, $H_{1}^{\prime}-1$ is an up-step of the bridge, where no tree is attached to, while $H_{1}-1$ is a down-step. As a consequence, if we modify $T_{\infty}$ by attaching an independent tree with law $\rho_{0}$ to $H_{1}^{\prime}-1$, the whole process $\left(\mathrm{b}_{\infty}, T_{\infty}\right)$ has the same law on $\left[0, H_{1}\right]$ as on $\left[H_{1}^{\prime}, 0\right]$. Thus, for every $m \in \mathbb{Z}_{+}$,

$$
\mathbb{P}\left(\Delta_{0}<m\right)=\mathbb{P}\left(\Delta_{0}^{\prime}<m\right) h(m),
$$

which gives from the first part of the proposition that for every $m \in \mathbb{N}$,

$$
\mathbb{P}\left(\Delta_{0}^{\prime} \geq m\right)=\frac{1}{m+3} .
$$

This concludes the proof of Proposition 3.1.

Remark 3.3. Note that as an intermediate step in the proof of Proposition 3.1, we explicitly compute the distribution of a minimal label in a well-labeled tree $(\tau, \ell)$ with law $\rho_{0}$, cf. Display (3.2). As it was pointed out to us by the referee, the calculation of $h(m)$ was already performed in Chassaing and Durhuus (2006, Proposition 2.4). In Curien et al. (2013, Lemma 12), it is (only) shown that the tail distribution behaves asymptotically like $2 / m^{2}$ as $m$ tends to infinity. The methods of Curien et al. (2013) rely on the fact that the label function $\ell$ has its continuous analog in the so-called Brownian snake. We stress that for our purpose, the asymptotic tail behavior of the minimal label of $(\tau, \ell)$ would not provide enough information, see Remark 3.5 below.

We let $Q_{\infty}^{\infty}=\Phi\left(\left(\mathrm{b}_{\infty}, T_{\infty}\right)\right)$ be the UIHPQ defined in terms of a uniform infinite treed bridge $\left(\mathrm{b}_{\infty}, T_{\infty}\right)$. Recall the identification of $\mathbb{Z}$ with $\partial Q_{\infty}^{\infty}$ via the function $\varphi$. Our presentation is now similar to that of Curien et al. (2013, Section 3.2.2). From now on, $\gamma_{\max }$ will denote the maximal geodesic in the UIHPQ emanating from the root $\varrho$. By construction of the Bouttier-Di Francesco-Guitter mapping and by definition of $\gamma_{\max }$, a vertex $\varphi(j) \in \partial Q_{\infty}^{\infty}$ for $j \in \mathbb{Z}_{+}$is hit by $\gamma_{\max }$ if and only if it is incident to the first (real) corner in contour order starting from $c_{0}$ with label $\ell_{\infty}(\varphi(j))$, i.e., if and only if

$$
\min \left\{\ell_{\infty, i}(v): v \in V\left(T_{\infty}(i)\right), i \in \mathrm{DS}\left(\mathrm{b}_{\infty}\right), 0 \leq i \leq j-1\right\}>\mathrm{b}_{\infty}(j),
$$

where $\ell_{\infty, i}$ denotes the labeling of $T_{\infty}(i)$. In particular, if we introduce the set of intersection times of the maximal geodesic with the right boundary of the UIHPQ,

$$
\mathcal{R}_{+}=\left\{j \in \mathbb{Z}_{+}: \gamma_{\max }(j) \in \varphi\left(\mathbb{Z}_{+}\right)\right\},
$$


we have

$$
\mathcal{R}_{+}=\mathbb{Z}_{+} \backslash \bigcup_{j \geq 0}\left(j, j+\Delta_{j}\right] .
$$

See Figure 3.6 for an illustration. It follows from the last display that $\mathcal{R}_{+}$can be represented as the set $\left\{G_{0}+G_{1}+\cdots+G_{n}: n \in \mathbb{Z}_{+}\right\}$, where $G_{0}=0$, and $\left(G_{i}: i \in \mathbb{N}\right)$ is a sequence of i.i.d. variables with

$$
G_{1}=\inf \left\{i>0: \max \left\{j+\Delta_{j}: 0 \leq j \leq i-1\right\}<i\right\} .
$$

In particular, $\mathcal{R}_{+}$is a discrete regenerative set, and the renewal theorem shows that the asymptotic frequency of $\mathcal{R}_{+}$is given by

$$
\left|\mathcal{R}_{+}\right|=\lim _{n \rightarrow \infty} \frac{\# \mathcal{R}_{+} \cap\{1, \ldots, n\}}{n}=\frac{1}{\mathbb{E}\left[G_{1}\right]} .
$$

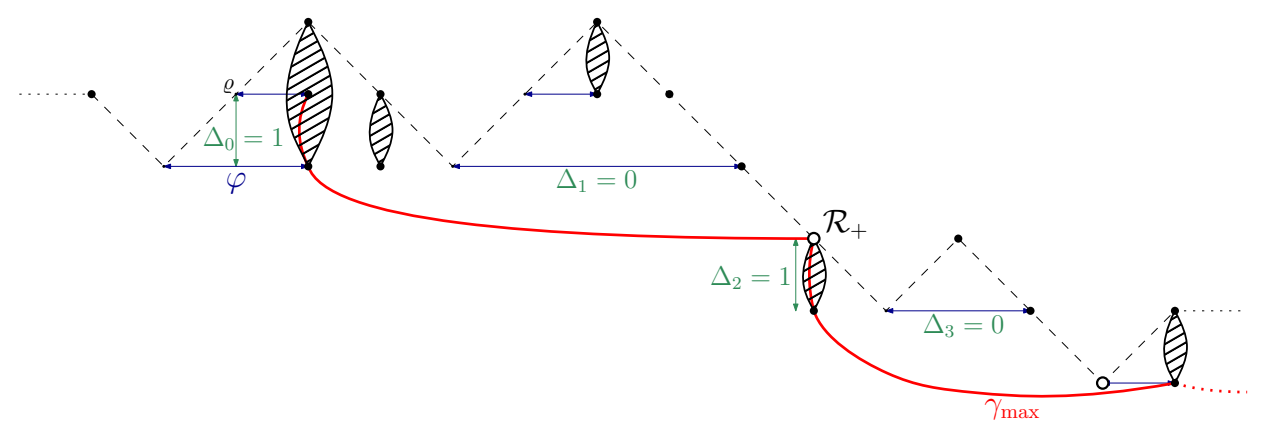

FIGURE 3.6. Alternative representation of the UIHPQ and its maximal geodesic $\gamma_{\max }$ as depicted in Figure 2.3. (Trees are represented by the striped almonds, whose lower endpoints indicate the minimal label in the corresponding tree.)

We will also study the set of intersection times of the maximal geodesic with the left part of the boundary,

$$
\mathcal{R}_{-}=\left\{j \in \mathbb{Z}_{+}: \gamma_{\max }(j) \in \varphi\left(\mathbb{Z}_{-}\right)\right\} .
$$

Using again the construction of the UIHPQ via the Bouttier-Di Francesco-Guitter mapping, we can express this set as

$$
\mathcal{R}_{-}=\mathbb{Z}_{+} \backslash \bigcup_{j \geq 0}\left(j, j+\Delta_{j}^{\prime}\right] .
$$

Similarly to $\mathcal{R}_{+}$, we have $\mathcal{R}_{-}=\left\{G_{0}^{\prime}+G_{1}^{\prime}+\cdots+G_{n}^{\prime}: n \in \mathbb{Z}_{+}\right\}$, where again $G_{0}^{\prime}=0$, and $\left(G_{i}^{\prime}: i \in \mathbb{N}\right)$ is an i.i.d. family of random variables specified by

$$
G_{1}^{\prime}=\inf \left\{i>0: \max \left\{j+\Delta_{j}^{\prime}: 0 \leq j \leq i-1\right\}<i\right\} .
$$

Note that $\left(G_{i}^{\prime}: i \in \mathbb{N}\right)$ is also independent of $\left(G_{i}: i \in \mathbb{N}\right)$. Indices $j \in \mathcal{R}_{-}$correspond to (certain) up-steps of the bridge and thus to phantom vertices. Then, the associated vertex $\varphi(j)$ is incident to the first (real) corner in contour order starting from $c_{0}$ with label $\ell_{\infty}(\varphi(j))$ and is therefore visited by the maximal geodesic.

We now formulate the key proposition of this paper. 
Proposition 3.4. We have for $i \in \mathbb{N}$,

$$
\mathbb{P}\left(i \in \mathcal{R}_{+}\right)=\frac{1}{i+1}, \quad \text { and } \quad \mathbb{P}\left(i \in \mathcal{R}_{-}\right)=\frac{3}{i+3} .
$$

Also, almost surely, both $\mathcal{R}_{+}$and $\mathcal{R}_{-}$are infinite sets, and the maximal geodesic $\gamma_{\max }$ hits the left as well as the right part of the boundary of the UIHPQ infinitely many times. However, this happens with asymptotic frequency zero: $\left|\mathcal{R}_{+}\right|=0$ and $\left|\mathcal{R}_{-}\right|=0$ almost surely.

Proof: The arguments for $\mathcal{R}_{+}$and $\mathcal{R}_{-}$are entirely similar. Let us first consider $\mathcal{R}_{+}$. By Proposition 3.1 in the last equation, we have for $i \in \mathbb{N}$

$$
\begin{aligned}
\mathbb{P}\left(i \in \mathcal{R}_{+}\right) & =\mathbb{P}\left(\max \left\{j+\Delta_{j}: 0 \leq j \leq i-1\right\}<i\right) \\
& =\prod_{j=0}^{i-1}\left(1-\mathbb{P}\left(\Delta_{0} \geq i-j\right)\right)=\prod_{j=1}^{i}\left(1-\mathbb{P}\left(\Delta_{0} \geq j\right)\right) \\
& =\exp \left(\sum_{j=1}^{i} \ln \left(1-\frac{1}{j+1}\right)\right)=\frac{1}{i+1} .
\end{aligned}
$$

We deduce from the last display that

$$
\mathbb{E}\left[\# \mathcal{R}_{+}\right]=\sum_{i=0}^{\infty} \mathbb{P}\left(i \in \mathcal{R}_{+}\right)=\infty
$$

From this, we readily infer that $\# \mathcal{R}_{+}=\infty$ almost surely: Indeed, if the contrary were true, then necessarily $G_{1}=\infty$ with some probability $\alpha>0$. However, then the number of points in $\mathcal{R}_{+}$different from 0 is geometrically distributed with parameter $\alpha$, a contradiction to $\mathbb{E}\left[\# \mathcal{R}_{+}\right]=\infty$. The fact that $\left|\mathcal{R}_{+}\right|=0$ follows from (3.7) and Proposition 3.1. Concerning $\mathcal{R}_{-}$, we simply have to replace $\Delta_{0}$ by $\Delta_{0}^{\prime}$ in the above argumentation. An application of Proposition 3.1 shows $\mathbb{P}\left(i \in \mathcal{R}_{-}\right)=3 /(i+3)$, and the remaining statements for $\mathcal{R}_{-}$follow from the same reasoning as above.

Albeit being infinite, the sets $\mathcal{R}_{+}$and $\mathcal{R}_{-}$are rather sparse. We will make this more precise in Section 4.

Remark 3.5. The last proposition should be compared with Proposition 15 of Curien et al. (2013). Proposition 3.1 has its counterpart in Lemma 14 of Curien et al. (2013), where it is shown that the quantity corresponding to $\mathbb{P}\left(\Delta_{0} \geq m\right)$ behaves asymptotically like $2 / m$ for $m$ tending to infinity. The multiplicative factor being larger than 1, this implies in the context considered there that the number of intersections between the maximal geodesic and the spine of the UIPQ is finite almost surely. Here, in the setting of the UIHPQ, we find an exact formula for $\mathbb{P}\left(\Delta_{0} \geq m\right)$, which came somewhat as a surprise and is the key observation that leads to Proposition 3.4. We emphasize that an equivalent of the form $\mathbb{P}\left(\Delta_{0} \geq m\right) \sim$ $1 / m$ would not be sufficient to deduce that $\mathcal{R}_{+}$is an infinite set, and the same for $\mathcal{R}_{-}$.

For the intersection of the independent regenerative sets $\mathcal{R}_{+}$and $\mathcal{R}_{-}$, we have for $i \in \mathbb{Z}_{+}$

$$
\mathbb{P}\left(i \in \mathcal{R}_{+} \cap \mathcal{R}_{-}\right)=\frac{3}{(i+1)(i+3)},
$$

and with arguments similar to those in the proof of Proposition 3.4, we get that the left and right boundary of the UIHPQ intersect finitely many times. Actually, we 
have here obtained a new proof of the fact shown in Curien and Miermont (2015) that the UIHPQ contains a well-defined core, that is an infinite submap homeomorphic to the half-plane. In Curien and Miermont (2015), the well-definedness of the core was obtained by a limiting argument, starting from an infinite quadrangulation with a simple boundary of a finite (randomized) size, while we prove this result directly in terms of the UIHPQ.

Note that since any maximal geodesic finally coincides with $\gamma_{\max }$, Proposition 3.4 implies that any maximal geodesic has infinitely many intersection points with the left and right part of the boundary of the UIHPQ. We now prove that all geodesic rays in the UIHPQ are proper. Theorem 1.1 will then readily follow. The following result was already established in Proposition 4.8 of Caraceni and Curien (2015) for geodesic rays started from the root vertex, by similar but different arguments.

Corollary 3.6 (see Proposition 4.8 of Caraceni and Curien, 2015). Almost surely, all geodesics rays in the UIHPQ $Q_{\infty}^{\infty}=\Phi\left(\left(\mathrm{b}_{\infty}, T_{\infty}\right)\right)$ are proper.

Proof: Here, we propose a simple proof that uses the result of Proposition 3.4. Let $\eta$ be an infinite self-avoiding path in $Q_{\infty}^{\infty}$. Since by the above proposition, the maximal geodesic $\gamma_{\max }$ intersects the left and right boundary infinitely often, the path $\eta$ also intersects $\gamma_{\max }$ infinitely often, as indicated by Figure 3.7.

Let $\gamma$ be a geodesic ray in $Q_{\infty}^{\infty}$. To simplify notation, we assume that $\gamma$ starts at the root $\varrho$ (if not, one should consider the maximal geodesic started from $\gamma(0)$ ). The above remark applied to $\eta=\gamma$ shows that $\gamma$ and $\gamma_{\max }$ intersect infinitely many times. Let $\left(u_{i}: i \in \mathbb{Z}_{+}\right)$be the sequence of vertices at which $\gamma$ and $\gamma_{\text {max }}$ intersect, with $u_{0}=\varrho$ and such that $u_{i}$ is visited before $u_{j}$ if $i<j$. Then, for every $i \in \mathbb{Z}_{+}$, by definition of the maximal geodesic,

$$
d_{\mathrm{gr}}\left(u_{i+1}, u_{i}\right)=\ell_{\infty}\left(u_{i}\right)-\ell_{\infty}\left(u_{i+1}\right) .
$$

Because labels differ at most by one between neighboring vertices of the map, the length of the segment of $\gamma$ between $u_{i}$ and $u_{i+1}$ is at least $\ell_{\infty}\left(u_{i}\right)-\ell_{\infty}\left(u_{i+1}\right)=$ $d_{\mathrm{gr}}\left(u_{i+1}, u_{i}\right)$. Therefore, equality must hold since $\gamma$ is a geodesic, and this implies that labels always decrease by one as $\gamma$ goes from $u_{i}$ to $u_{i+1}$, meaning that $\gamma$ is proper on this segment. This finishes the proof.

The proof of Theorem 1.1 is now an immediate consequence of our foregoing considerations.

Proof of Theorem 1.1: For the purpose of the proof, we will assume that the UIHPQ is given in terms of a uniform infinite treed bridge, $Q_{\infty}^{\infty}=\Phi\left(\left(\mathrm{b}_{\infty}, T_{\infty}\right)\right)$. Let $\gamma$ be a geodesic ray. By Corollary 3.6, we can assume that $\gamma$ is proper. Hence each edge of $\gamma$ connects a real corner of $\left(\mathrm{b}_{\infty}, T_{\infty}\right)$ to its successor. Now let $n_{0} \in \mathbb{Z}_{+}$ be the first instant when the maximal geodesic emanating from $v=\gamma(0)$ hits the left part of the boundary. We have seen above that $n_{0}$ is finite almost surely. By definition, $\gamma_{\max }^{v}$ always connects leftmost corners to their successors. In particular, the embedding of $\gamma$ in the upper half-plane (in terms of the Bouttier-Di FrancescoGuitter mapping) lies in between $\left(\gamma_{\max }^{v}(n): n \geq n_{0}\right)$ and the boundary of the map, see Figure 3.8. Otherwise said, vertices of the right part of the boundary which are visited by $\left(\gamma_{\max }^{v}(n): n \geq n_{0}\right)$ are also visited by any other proper geodesic started at $v$. Since $\gamma_{\max }^{v}$ coincides after a finite number of steps with $\gamma_{\max }$, the maximal geodesic started from the root $\varrho$, Proposition 3.4 concludes the proof. 


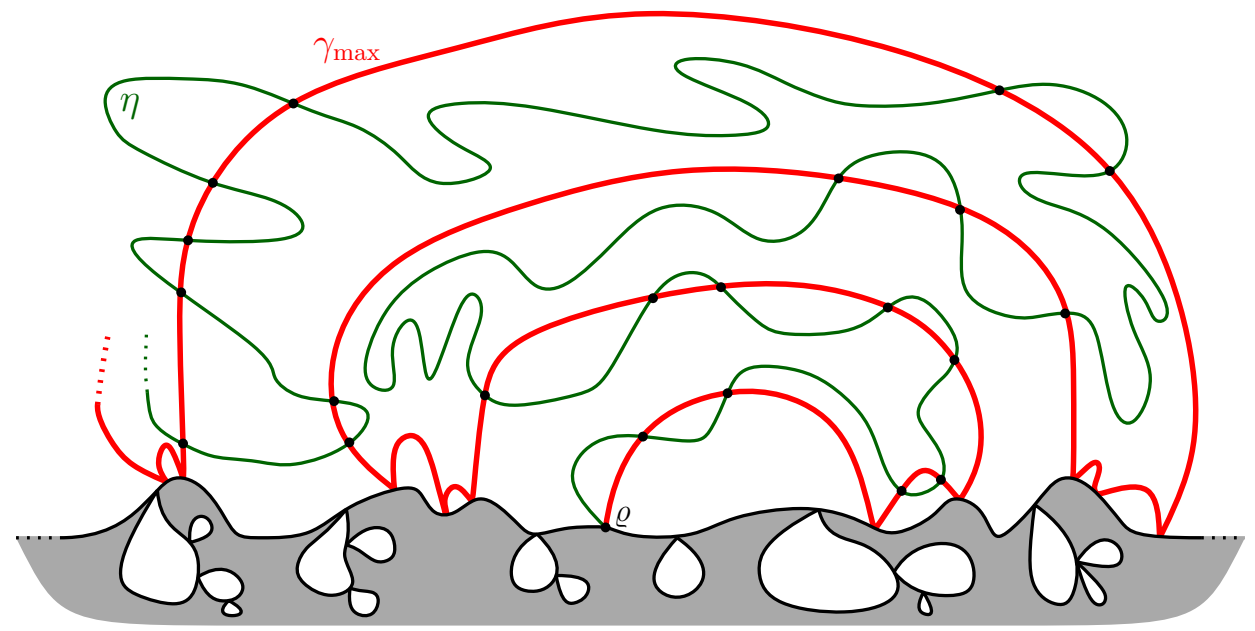

FigurE 3.7. The infinite path $\eta$ intersects $\gamma_{\max }$ infinitely many times.

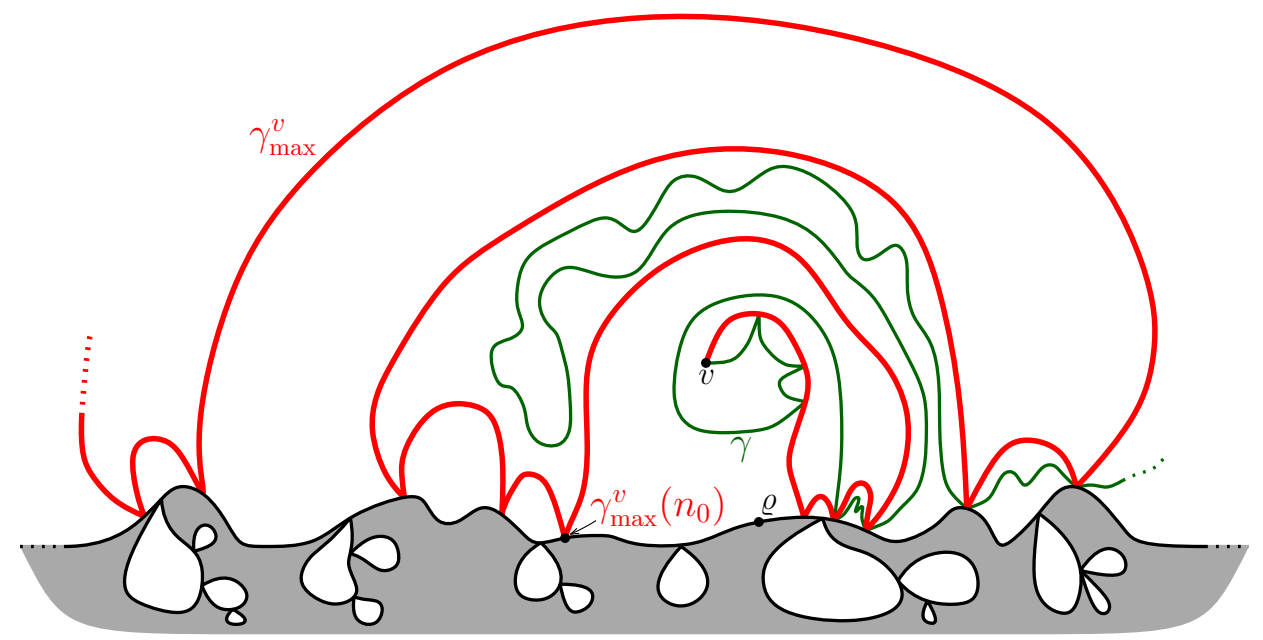

Figure 3.8. The geodesic $\gamma$ lies in between $\left(\gamma_{\max }^{v}(n): n \geq n_{0}\right)$ and the boundary of the map.

Corollary 3.7. Theorem 1.1 remains true if the UIHPQ is replaced by its analog with a simple boundary, the $\mathrm{UIHPQ}^{(s)}$.

Proof: We give only a sketch proof, since the statement is essentially a consequence of the pruning construction of the UIHPQ ${ }^{(s)}$ out of the UIHPQ, as explained in Curien and Miermont (2015) (see, in particular, Proposition 6 in this work). Roughly speaking, after removing the finite quadrangulations which hang off from the pinch-points of the boundary of the UIHPQ, a core consisting of a unique infinite quadrangulation with an infinite simple boundary remains, which has, after a rooting operation, the law of the UIHPQ ${ }^{(s)}$. Since geodesics started from the core of the UIHPQ do not visit the finite quadrangulations that are attached to the pinch-points 
of the boundary (the pinch-points would be visited twice), Theorem 1.1 applies to the $\mathrm{UIHPQ}^{(s)}$ as well.

\section{Sparseness of the intersections with the boundary}

From Theorem 1.1, we know that every geodesic ray in the UIHPQ hits the boundary infinitely many times. The goal of this section is to show that these hitting times and hitting points are, however, sparsely distributed, in a way that we will make precise in Proposition 4.3 below.

For that purpose, recall that the sets $\mathcal{R}_{+}$and $\mathcal{R}_{-}$of intersection times of the maximal geodesic with the right and left part of the boundary, respectively, admit the representation

$$
\mathcal{R}_{+}=\left\{G_{0}+G_{1}+\cdots+G_{n}: n \in \mathbb{Z}_{+}\right\}, \quad \mathcal{R}_{-}=\left\{G_{0}^{\prime}+G_{1}^{\prime}+\cdots+G_{n}^{\prime}: n \in \mathbb{Z}_{+}\right\},
$$

where $G_{0}=G_{0}^{\prime}=0$, and the families $\left(G_{i}: i \in \mathbb{N}\right)$ and $\left(G_{i}^{\prime}: i \in \mathbb{N}\right)$ consist of i.i.d. random variables specified by (3.6) and (3.8), respectively. We find the following asymptotic behavior.

Lemma 4.1. For $m$ tending to infinity, we have

$$
\mathbb{P}\left(G_{1}=m\right) \sim \frac{1}{m \ln ^{2} m}, \quad \mathbb{P}\left(G_{1}^{\prime}=m\right) \sim \frac{1}{3 m \ln ^{2} m} .
$$

Proof: We first look at $G_{1}$. For $n \in \mathbb{Z}_{+}$, let $u_{n}=\mathbb{P}\left(n \in \mathcal{R}_{+}\right), f_{n}=\mathbb{P}\left(G_{1}=\right.$ $n$ ). Note that $f_{0}=0$ and $u_{0}=1$. A classical decomposition (see, e.g., Section XIII.3 in Feller, 1968) of $u_{n}$ according to the smallest non-zero element in $\mathcal{R}_{+}$, i.e., according to the value of $G_{1}$, gives the recursive relation

$$
u_{n}=f_{1} u_{n-1}+f_{2} u_{n-2}+\cdots+f_{n} u_{0}, \quad n \in \mathbb{N} .
$$

For the generating functions $U(s)=\sum_{n \geq 0} u_{n} s^{n}$ and $F(s)=\sum_{n \geq 0} f_{n} s^{n}$, the last relation implies

$$
U(s)=\frac{1}{1-F(s)}, \quad|s|<1 .
$$

Using that $\mathbb{P}\left(n \in \mathcal{R}_{+}\right)=1 /(n+1)$, see Proposition 3.4, we obtain for $0<|s|<1$ the expression $U(s)=-(1 / s) \ln (1-s)$. Therefore,

$$
F(s)=1-s \ln ^{-1}\left(\frac{1}{1-s}\right), \quad|s|<1 .
$$

Standard singularity analysis, see, e.g., (24) on page 387 of Flajolet and Sedgewick (2009), yields the first claim. For $G_{1}^{\prime}$, we use that $\mathbb{P}\left(n \in \mathcal{R}_{-}\right)=3 /(n+3)$, see again Proposition 3.4. For the generating function $H(s)=\sum_{n \geq 0} \mathbb{P}\left(G_{1}^{\prime}=n\right) s^{n}$, this gives similarly to above the relation

$$
H(s)=1-\left(s^{3} / 3\right)\left(\ln \left(\frac{1}{1-s}\right)-s^{2} / 2-s\right)^{-1}, \quad|s|<1 .
$$

Since $1-H(s) \sim(1 / 3)(1-F(s))$ as $s \rightarrow 1$, an application of Flajolet and Sedgewick (2009, Theorem IV.4) finishes the proof of the second claim.

Remark 4.2. The above lemma should be compared with the asymptotics of the returns to zero of a recurrent two-dimensional random walk $S=\left(S_{n}: n \in \mathbb{Z}_{+}\right)$. For concreteness, let us assume that $S$ is the simple symmetric random walk on $\mathbb{Z}^{2}$ started from zero. Let $\mathcal{R}$ be the regenerative set of return times to zero of $S$. 
One has the representation $\mathcal{R}=\left\{G_{0}+G_{1}+\cdots+G_{n}: n \in \mathbb{Z}_{+}\right\}$, where $G_{0}=0$, and $\left(G_{i}: i \in \mathbb{N}\right)$ are the waiting times between two consecutive returns. Then, as $m \rightarrow \infty$, we get the asymptotics (Spitzer, 1976, Chapter III, Section 16, Example 1)

$$
\mathbb{P}(m \in \mathcal{R}) \sim \frac{1}{\pi m} \quad \text { and } \quad \mathbb{P}\left(G_{1}=m\right) \sim \frac{\pi}{m \ln ^{2} m} .
$$

Coming back to geodesics in the UIHPQ, we note that Lemma 4.1 gives precise quantitative information on the number of steps between two consecutive visits of the boundary by the maximal geodesic $\gamma_{\max }$. The distance measured along the boundary between two consecutive times of intersection is bounded from below by the number of steps of $\gamma_{\max }$ in between these times.

In the proof of Theorem 1.1, we have seen that any geodesic ray $\gamma$ is finally enclosed between $\gamma_{\max }$ and the boundary of the UIHPQ. A priori, this does not exclude the existence of a geodesic ray that visits the boundary with a much higher frequency than $\gamma_{\max }$. We will now argue that this is not the case.

In this regard, it is convenient to introduce the minimal geodesic in the UIHPQ emanating from the root $\varrho$. Given $\left(\mathrm{b}_{\infty}, T_{\infty}\right)$ and $v$ a real vertex of $\left(\mathrm{b}_{\infty}, T_{\infty}\right)$, we write $c^{(r)}(v)$ for the rightmost corner incident to $v$. Note that in the list of corners $\left(c_{i}\right)_{i \in \mathbb{Z}}$ as specified in Section 2.2.2, $c^{(r)}(v)$ appears as the last corner incident to $v$ (in the lexicographical order).

The minimal geodesic $\gamma_{\min }$ starting from $\varrho$ is then given by the chain of vertices $\gamma_{\min }(0)=\varrho$, and for $i \in \mathbb{N}$,

$$
\gamma_{\min }(i)=\mathcal{V}\left(\operatorname{succ}\left(c^{(r)}\left(\gamma_{\min }(i-1)\right)\right)\right) .
$$

The edge set of $\gamma_{\min }$ is given by the edges connecting $c^{(r)}\left(\gamma_{\min }(i)\right)$ to $c^{(r)}\left(\gamma_{\min }(i+1)\right)$ for $i \in \mathbb{Z}_{+}$.

Similarly to above, one defines for $\gamma_{\min }$ the (random) sets of intersection times with the right and left part of the boundary, respectively,

$$
\mathcal{R}_{+}^{\min }=\left\{j \in \mathbb{Z}_{+}: \gamma_{\min }(j) \in \varphi\left(\mathbb{Z}_{+}\right)\right\}, \quad \mathcal{R}_{-}^{\min }=\left\{j \in \mathbb{Z}_{+}: \gamma_{\min }(j) \in \varphi\left(\mathbb{Z}_{-}\right)\right\} .
$$

The following symmetry argument shows that the random set $\mathcal{R}_{+}^{\min }$ (defined in terms of $\gamma_{\text {min }}$ ) has the same law as $\mathcal{R}_{-}$(defined in terms of $\gamma_{\max }$ ). Consider the mapping that associates to a (possibly infinite) rooted planar map $\mathfrak{m}$ its "mirror" $\overleftarrow{\mathfrak{m}}$, which is obtained from applying a symmetry with respect to any line of the plane, and reversing the orientation of the root edge. This transformation is better understood by seeing a planar map as a gluing of polygons: Then, the map $\overleftarrow{\mathfrak{m}}$ is obtained by reversing the orientation of the polygons forming $\mathfrak{m}$, and that of the root edge. Now, it is seen that this transformation preserves the uniform measure on quadrangulations with a fixed size and perimeter, and thus the law of the UIHPQ. Finally, recall that the maximal and minimal geodesics started at the root vertex are also the leftmost and rightmost geodesics, respectively, and are thus exchanged by the "mirror" mapping. It follows that $\mathcal{R}_{+}^{\min }$ and $\mathcal{R}_{-}$have the same law, and, by the same symmetry argument, $\mathcal{R}_{-}^{\text {min }}$ has the same law as $\mathcal{R}_{+}$.

As a direct consequence of the way edges are drawn in the Bouttier-Di FrancescoGuitter construction of the UIHPQ, and of the fact that every geodesic ray is proper, see Corollary 3.6, we notice that any geodesic ray $\gamma$ lies finally in between $\gamma_{\max }$ and $\gamma_{\min }$. Indeed, this is the case from the first vertex on hit by $\gamma$ that is incident to a corner $c_{i}$ with $i \in \mathbb{Z}_{+}$. See Figure 4.9 for an illustration. From the constructions of 
$\gamma_{\max }$ and $\gamma_{\min }$, we see that $\mathcal{R}_{+}$is a subset of $\mathcal{R}_{+}^{\text {min }}$, and similarly $\mathcal{R}_{-}^{\text {min }}$ is a subset of $\mathcal{R}_{-}$.

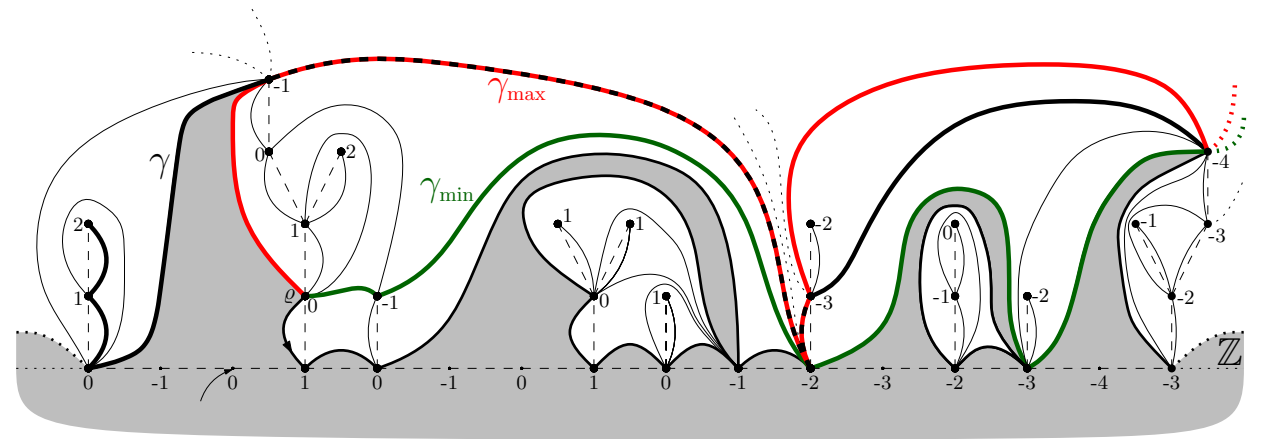

Figure 4.9. The geodesic $\gamma$ (black bold) started at the leftmost vertex labeled 2 is enclosed by $\gamma_{\min }$ (green bold) and $\gamma_{\max }$ (red bold) after it first hits the latter (at the topmost vertex labeled $-1)$.

We collect our observations in the following proposition, which should be read as an extension to Theorem 1.1. For simplicity, we restrict ourselves to geodesic rays emanating from the root vertex; see, however, the remark below the proposition.

Proposition 4.3. Almost surely, for any geodesic ray $\gamma=\left(\gamma(i): i \in \mathbb{Z}_{+}\right)$in the UIHPQ $Q_{\infty}^{\infty}=\Phi\left(\left(\mathrm{b}_{\infty}, T_{\infty}\right)\right)$ started from the root vertex, we have the inclusions

$$
\mathcal{R}_{+} \cup \mathcal{R}_{-}^{\min } \subseteq\left\{i \in \mathbb{Z}_{+}: \gamma(i) \in \partial Q_{\infty}^{\infty}\right\} \subseteq \mathcal{R}_{+}^{\min } \cup \mathcal{R}_{-} .
$$

The random sets $\mathcal{R}_{+}$and $\mathcal{R}_{-}^{\text {min }}$ (as well as $\mathcal{R}_{+}^{\min }$ and $\mathcal{R}_{-}$) have the same law. The distance $\delta$ between two consecutive times in $\mathcal{R}_{+}$(or $\mathcal{R}_{-}^{\min }$ ) exhibits the tail behavior $\mathbb{P}(\delta>m) \sim 1 / \ln m$ as $m \rightarrow \infty$, whereas the distance $\delta^{\prime}$ between two consecutive times in $\mathcal{R}_{+}^{\min }$ (or $\left.\mathcal{R}_{-}\right)$satisfies $\mathbb{P}\left(\delta^{\prime}>m\right) \sim 1 /(3 \ln m)$.

Remark 4.4. Let $\gamma=\left(\gamma(i): i \in \mathbb{Z}_{+}\right)$be any geodesic ray in the UIHPQ (not necessarily started from the root vertex), and let $v$ be the first vertex to the right of the root $\varrho$ which is hit by both $\gamma$ and $\gamma_{\max }$. Let $n, n^{\prime} \in \mathbb{Z}_{+}$such that $\gamma(n)=$ $\gamma_{\max }\left(n^{\prime}\right)=v$, and set $j=n-n^{\prime}$. Now consider the shifted geodesic $\gamma_{j}(i)=\gamma(i+j)$, $i \geq \max \{0,-j\}$. On the event of full probability where $\gamma, \gamma_{\max }$ and $\gamma_{\min }$ are proper, we have the inclusions

$$
\left(\mathcal{R}_{+} \cup \mathcal{R}_{-}^{\min }\right) \backslash\left\{0, \ldots, n^{\prime}\right\} \subseteq\left\{i \geq \max \{0,-j\}: \gamma_{j}(i) \in \partial Q_{\infty}^{\infty}\right\} \subseteq \mathcal{R}_{+}^{\min } \cup \mathcal{R}_{-} .
$$

\section{Extension to the uniform infinite half-planar triangulation and further remarks}

The uniform infinite half-planar triangulation UIHPT is an infinite triangulation of the half-plane. A variation with a simple boundary (i.e., the triangular analog to the UIHPQ ${ }^{(s)}$ ) was introduced by Angel (2005).

In this part, we will argue that the intersection times with the boundary of geodesics in the UIHPT behave in way comparable to that in the UIHPQ. More precisely, it turns out that the right part of the boundary is hit by the maximal 
geodesic started from the root with exactly the same frequency as in the UIHPQ, whereas the distribution of the hitting times of the left part of the boundary undergoes a slight change.

In order to avoid too much repetition, we will not treat the case of the UIHPT in full detail. We will rather argue that the strategy developed for the UIHPQ applies to the UIHPT as well, and then sketch how the computations have to be modified. Our discussion will therefore lack a certain rigor, but should enable the reader to fill in the remaining details. In order to make a clear distinction to the UIHPQ, some of our quantities considered in this section will be decorated with the tilde sign.

Triangulations, or more generally (rooted and pointed) planar maps with prescribed face valences, can be encoded in terms of labeled trees called mobiles, see Bouttier et al. (2004). Let us briefly recall the encoding: First, label each vertex of the map by its distance from the pointed vertex minus the distance from the pointed vertex to the origin of the root edge. Put a new vertex without label in the center of each face. Now walk around each face $F$ in the clockwise order, and look at each of its incident edges. If for an edge $e$, the label decreases by 1 when walking clockwise around $F$, then connect the endpoint of $e$ with the larger label to the (unlabeled) vertex in the middle of $F$. If the labels of the endpoints of $e$ are both equal to $n$, say, add a flagged vertex with flag $n$ in the middle of $e$ and connect the flagged vertex with two new edges to the two central vertices of the faces incident to $e$. In the third case, that is, for edges where the labels increase when walking around the face $F$, do nothing. See Figure 5.10. By removing all the original edges of the map together with the pointed vertex, one obtains a mobile, i.e., a plane tree with three types of vertices: labeled and unlabeled vertices, and flagged vertices.

Note that by construction, flagged vertices have degree 2 , and unlabeled vertices are in one-to-one correspondence with the faces of the map. Moreover, the degree of the corresponding face equals twice the number of labeled vertices plus the number of flagged vertices that are connected to the unlabeled vertex in the mobile. In particular, an unlabeled vertex associated to a triangular face has either three flagged vertices or a flagged vertex and a labeled vertex incident to it.
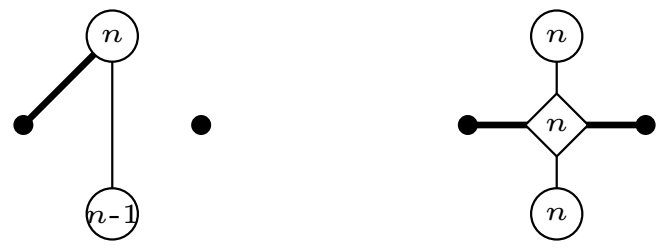

FiguRE 5.10. The construction of a mobile. The black dots represent unlabeled vertices of the mobile. They are put in the centers of the faces of the map. On the left, the bold line represents a mobile edge associated to an edge of the map, which connects a vertex labeled $n$ to a vertex labeled $n-1$. On the right, the bold line represents a mobile edge associated to an edge connecting two vertices with label $n$. The flagged vertex is represented by a lozenge and receives label $n$, too. 

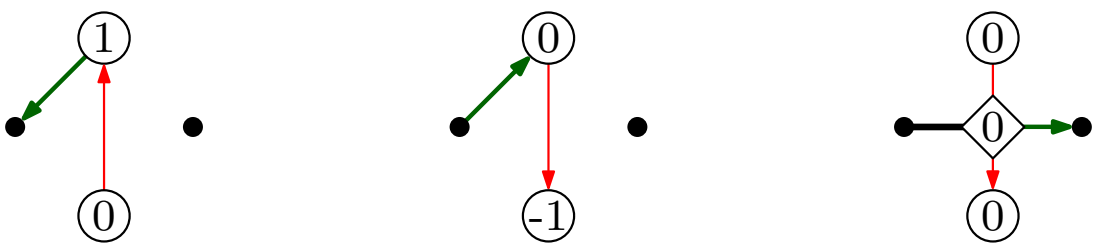

Figure 5.11. The rooting convention. The red arrow represents the root edge of the map, and the green bold arrow is the associated root edge of the mobile.

The root edge of a planar map allows to distinguish a root edge in the mobile, as depicted in Figure 5.11. If the root edge of the map connects two vertices with label 0 , see the right most case in Figure 5.11, it is convenient to regard the encoding mobile as a pair of half-mobiles with root flag 0 each, i.e., mobiles which have one distinguished flagged vertex of degree 1 called the root flag, which receives label 0 . There is a bijection between rooted pointed planar maps on the one hand and rooted mobiles and pairs of half-mobiles on the other hand. We refer to Bouttier et al. (2004) and Bouttier and Guitter (2012) for more details.

In terms of generating functions, prescribing the number of faces of a certain degree $k$ amounts to attach a weight to each face of degree $k$. For our purpose, we now specialize in triangulations corresponding to the critical weight sequence $g_{k}=$ $g_{3, \mathrm{cr}} \delta_{3}(k)$, where $g_{3, \mathrm{cr}}=2^{-1} 3^{-3 / 4}$, see, e.g., Miermont (2006). In this regard, let $R_{m}$ (or $S_{m}$ ) denote the corresponding generating function of rooted mobiles (or halfmobiles) with root label (or root flag) 0, which have their labels all strictly larger than $-m$ and their flags all larger or equal to $-m$, cf. Bouttier and Guitter (2012). Letting $R=\lim _{m \rightarrow \infty} R_{m}$ and $S=\lim _{m \rightarrow \infty} S_{m}$, an analysis of (6.2) in Bouttier and Guitter (2012) shows that $R=\sqrt{3}$ and $S=3^{1 / 4}(\sqrt{3}-1)$, but this will be of no importance here. Note that $R$ and $S$ are the partition functions for rooted mobiles with root label 0 and half-mobiles with root flag 0 , respectively, subject to $g_{k}=g_{3, \mathrm{cr}} \delta_{3}(k)$.

In order to motivate our construction of the UIHPT, let us first consider rooted pointed triangulations with a boundary of perimeter $n \in \mathbb{Z}_{+}$. This means that all faces except the root face are triangles, the root face being incident to $n$ edges (loops and multiple edges are allowed). We choose such a triangulation $\mathfrak{m}$ according to the Boltzmann law $\rho(\mathfrak{m})=g_{3, \mathrm{cr}}^{\# F(\mathfrak{m})} / Z$, where $F(\mathfrak{m})$ denotes the set of faces of $\mathfrak{m}$ without the root face (which receives no weight), and $Z$ is the normalizing partition function. Denote by $d$ the distance between the pointed vertex of $\mathfrak{m}$ and the origin of the root edge. Following Section 2.4 of Bouttier et al. (2004), we associate to the map a (random) path $\left(X^{[n]}(i): 0 \leq i \leq n\right)$ that encodes the clockwise sequence of distances minus $d$ between the pointed vertex of the map and the vertices incident to the root face, with $X^{[n]}(0)$ given by the origin of the root edge (so that $X^{[n]}(0)=0$ ).

We decompose the associated mobile around the unlabeled vertex $v$ lying in the center of the root face of the map. Then each down- or level-step of $X^{[n]}$ corresponds to a labeled or a flagged vertex, respectively, which is connected to $v$ by an edge, see Figure 5.10. By removing $v$ and its incident edges, one obtains a sequence of rooted mobiles and half-mobiles. More precisely, a down-step $i$ of $X^{[n]}$ corresponds to a rooted mobile with root label $X^{[n]}(i)$, while a level-step $i$ of $X^{[n]}$, that is, an $i$ 
with $X^{[n]}(i+1)=X^{[n]}(i)$, corresponds to a half-mobile with root flag $X^{[n]}(i)$. This decomposition is bijective. Letting $n$ grow, this incites us to define the following two-sided random walk. Let $C=2 \sqrt{R}+S$, and consider $\tilde{\mathrm{b}}_{\infty}=\left(\tilde{\mathrm{b}}_{\infty}(i): i \in \mathbb{Z}\right)$ with $\tilde{\mathrm{b}}_{\infty}(0)=0$, such that the increments $\left(\tilde{\mathrm{b}}_{\infty}(i+1)-\tilde{\mathrm{b}}_{\infty}(i): i \in \mathbb{Z}_{+}\right)$are i.i.d. with law

$$
\mathbb{P}\left(\tilde{\mathrm{b}}_{\infty}(i+1)-\tilde{\mathrm{b}}_{\infty}(i)= \pm 1\right)=\frac{\sqrt{R}}{C}, \quad \mathbb{P}\left(\tilde{\mathrm{b}}_{\infty}(i+1)-\tilde{\mathrm{b}}_{\infty}(i)=0\right)=\frac{S}{C},
$$

and $\left(\tilde{\mathrm{b}}_{\infty}(i): i \in \mathbb{Z}_{-}\right)$is an i.i.d. copy of $\left(\tilde{\mathrm{b}}_{\infty}(i): i \in \mathbb{Z}_{+}\right)$. One can show that for fixed $\ell \in \mathbb{N}$, there is the convergence

$$
\left(X^{[n]}([i]):-\ell \leq i \leq \ell\right) \underset{n \rightarrow \infty}{\stackrel{(d)}{\longrightarrow}}\left(\tilde{\mathrm{b}}_{\infty}(i):-\ell \leq i \leq \ell\right),
$$

with $[i]$ denoting the representative of $i$ modulo $n$ in $\{0, \ldots, n-1\}$.

We proceed now similarly to the construction of the UIHPQ: Conditionally on $\tilde{\mathrm{b}}_{\infty}$, we identify $\tilde{\mathrm{b}}_{\infty}$ with $\mathbb{Z}$ equipped with the labels $\left(\tilde{\mathrm{b}}_{\infty}(i): i \in \mathbb{Z}\right)$, and graft independently to each down-step $i \in \mathrm{DS}\left(\tilde{\mathrm{b}}_{\infty}\right)$ a mobile $\theta$ in the upper half-plane with root label $\tilde{b}_{\infty}(i)$, distributed according to the Boltzmann measure $\rho^{(R)}(\theta)=$ $g_{3, \mathrm{cr}}^{\# \bullet(\theta)} / R$ (where $\bullet(\theta)$ denotes the set of unlabeled vertices of $\theta$ ). Moreover, writing $\mathrm{LS}\left(\tilde{\mathrm{b}}_{\infty}\right)$ for the set of level-steps of $\tilde{\mathrm{b}}_{\infty}$, we graft to each $i \in \mathrm{LS}\left(\tilde{\mathrm{b}}_{\infty}\right)$ independently a half-mobile $\theta^{\prime}$ with root flag $\tilde{\mathrm{b}}_{\infty}(i)$, distributed according to $\left.\rho^{(S)}\left(\theta^{\prime}\right)=g_{3, \mathrm{cr}}^{\# \bullet} / \theta^{\prime}\right) / S$. We obtain what we call a uniform infinite mobile bridge $\left(\tilde{\mathrm{b}}_{\infty}, \tilde{T}_{\infty}\right)$, where $\tilde{T}_{\infty}$ is now a collection of independent mobiles and half-mobiles associated to the downand level-steps of $\tilde{b}_{\infty}$, respectively.

Each realization of $\left(\tilde{b}_{\infty}, \tilde{T}_{\infty}\right)$ is naturally embedded in the upper-half plane, similarly to the description in Section 2.2.2. Recall that mobiles and half-mobiles come with three types of vertices. We call here a labeled vertex of a mobile or a half-mobile a real vertex, and a real corner (of the embedding) is a corner in the upper half-plane incident to a real vertex. Note that flagged vertices are not real vertices.

We write $\left(c_{i}\right)_{i \in \mathbb{Z}}$ for the sequence of real corners in the left-to-right order, again with $c_{0}$ being the leftmost corner incident to the root vertex. As in the construction of the UIHPQ, we now connect each real corner $c_{i}$ to its successor, that is the first corner among $c_{i+1}, c_{i+2}, \ldots$ with label $\ell\left(c_{i}\right)-1$. Additionally, we connect both corners of the flagged vertices to the corresponding next real corner in the contour order with the same label. See Figure 5.12 for an illustration.

We finally erase the unlabeled vertices and the flagged vertices, interpreting the two outgoing arcs from a flagged vertex which we added as a single edge. We also erase all the edges and non-real vertices that stem from the representation of $\left(\tilde{\mathrm{b}}_{\infty}, \tilde{T}_{\infty}\right)$ in the plane. We obtain what we call the uniform infinite half-planar triangulation UIHPT. The bi-infinite line $\mathbb{Z}$ can again be identified with the boundary of the UIHPT. In particular, it makes sense to speak of the left or right part of the boundary. We root the UIHPT according to the convention described in Section 2.2.3.

Remark 5.1. We stress that the above construction does not make use of the particular form of the weight sequence and can therefore be carried through for maps corresponding to other critical or sub-critical Boltzmann weights. For the choice $g_{k}=(1 / 12) \delta_{4}(k)$, we rediscover the construction of the UIHPQ as described in 
Section 2.2.2. Note that for bipartite maps, we have $S=0$, i.e., there are no half-mobiles.

We may now define maximal (and minimal) geodesics in the UIHPT. Note that vertices of the UIHPT correspond to real vertices of the encoding. Analogously to the UIHPQ, the maximal geodesic started at vertex $v$ is given by the infinite chain of vertices which are incident to the iterated successors of the leftmost real corner $c$ belonging to $v$.

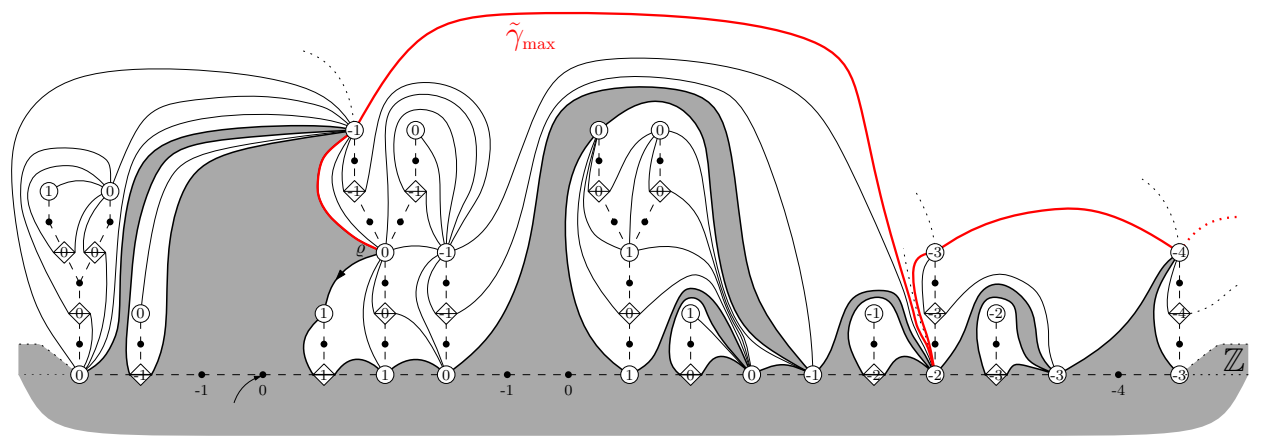

FIGURE 5.12. The construction of the UIHPT from an infinite mobile bridge, with its maximal geodesic $\tilde{\gamma}_{\max }$.

Similarly, by starting from the rightmost corner, we define the minimal geodesic emanating from $v$, and we write $\tilde{\gamma}_{\max }\left(\right.$ or $\left.\tilde{\gamma}_{\min }\right)$ for the maximal (or minimal) geodesic starting from the root vertex. Moreover, we let $\tilde{\mathcal{R}}_{+}$and $\tilde{\mathcal{R}}_{-}$(or $\tilde{\mathcal{R}}_{+}^{\text {min }}$ and $\tilde{\mathcal{R}}_{-}^{\text {min }}$ ) denote the set of intersection times of $\tilde{\gamma}_{\max }\left(\right.$ or $\tilde{\gamma}_{\min }$ ) with the right and left part of the boundary, respectively.

For characterizing $\tilde{\mathcal{R}}_{+}$and $\tilde{\mathcal{R}}_{-}$as regenerative sets, we may argue as in the case of the UIHPQ. For $j \in \mathbb{Z}_{+}$, let

$$
\tilde{\Delta}_{j}=\max _{i \in \operatorname{DS}\left(\tilde{\mathrm{b}}_{\infty}\right) \cup \operatorname{LS}\left(\tilde{\mathrm{b}}_{\infty}\right) \cap\left[H_{j}, H_{j+1}\right)}-\left(\min _{u \in V\left(\tilde{T}_{\infty}(i)\right)} \ell_{i}(u)+j\right) .
$$

Here, $H_{j}=H_{j}\left(\tilde{\mathrm{b}}_{\infty}\right)$, and in hopefully obvious notation, $\tilde{T}_{\infty}(i)$ is the mobile (in the case $i \in \mathrm{DS}\left(\tilde{\mathrm{b}}_{\infty}\right)$ ) or half-mobile (in the case $i \in \mathrm{LS}\left(\tilde{\mathrm{b}}_{\infty}\right)$ ) grafted to the vertex $i$, and $\ell_{i}(u)$ for $u \in V\left(\tilde{T}_{\infty}(i)\right)$ represents its label. By replacing $H_{j}$ with $H_{j}^{\prime}$, we define $\tilde{\Delta}_{j}^{\prime}$ in a similar fashion.

Proposition 5.2. We have for $m \in \mathbb{N}$,

$$
\mathbb{P}\left(\tilde{\Delta}_{0} \geq m\right)=\frac{1}{m+1}, \quad \text { and } \quad \mathbb{P}\left(\tilde{\Delta}_{0}^{\prime} \geq m\right)=\frac{1}{m+2}
$$


Proof: We first look at $\tilde{\Delta}_{0}$. Let $m \in \mathbb{N}$. Put $\tilde{g}(m)=1-\mathbb{P}\left(\tilde{\Delta}_{0} \geq m\right)$. The arch decomposition corresponding to (3.1) reads

$$
\begin{aligned}
\tilde{g}(m) & =\sum_{k=0}^{\infty}\left(\sum_{k^{\prime}=0}^{\infty}\left(\frac{S_{m}}{C}\right)^{k^{\prime}} \frac{\sqrt{R}}{C} \tilde{g}(m+1)\right)^{k} \sum_{\ell=0}^{\infty}\left(\frac{S_{m}}{C}\right)^{\ell} \frac{\sqrt{R}}{C} \frac{R_{m}}{R} \\
& =\frac{1}{1-\left(\frac{1}{1-\frac{S_{m}}{C}} \frac{\sqrt{R}}{C}\right) \tilde{g}(m+1)} \frac{1}{1-\frac{S_{m}}{c}} \frac{\sqrt{R}}{C} \frac{R_{m}}{R} ;
\end{aligned}
$$

see Figure 5.13. The formula for $\tilde{g}$ is equivalent to

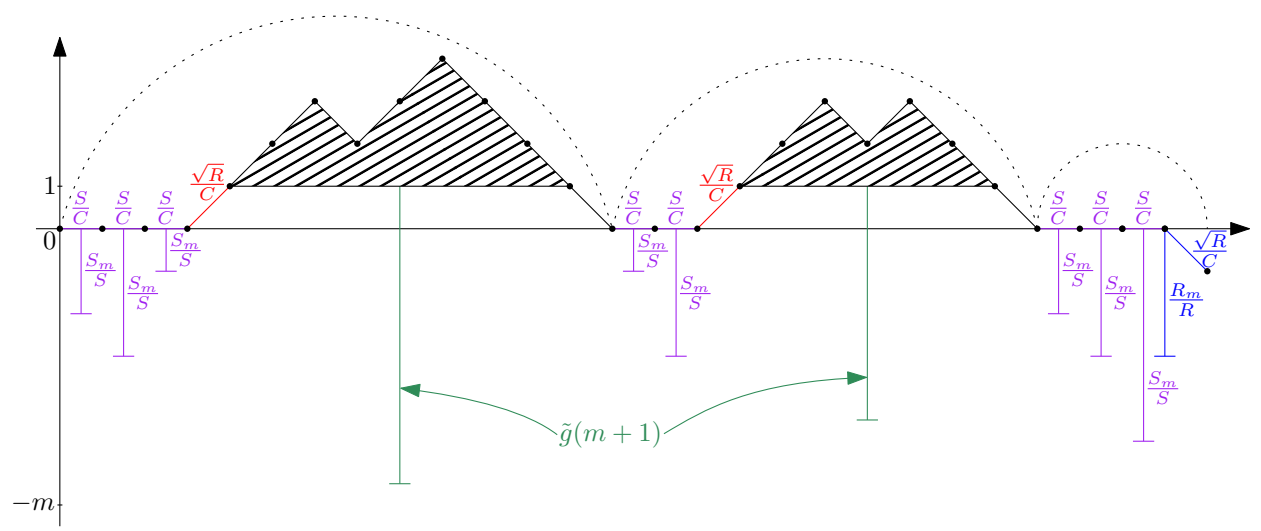

Figure 5.13. The decomposition of the probability $\tilde{g}(m)$.

$$
\tilde{g}(m)\left(\frac{C}{\sqrt{R}}-\frac{S_{m}}{S} \frac{S}{\sqrt{R}}-\tilde{g}(m+1)\right)=\frac{R_{m}}{R} .
$$

We note along the way that the last expression is universal, in the sense that it does not depend on the particular choice of the Boltzmann weights $\left(g_{k}\right)_{k \in \mathbb{N}}$.

Back to the triangular case, by letting $y \uparrow 1$ in (6.8) of Bouttier and Guitter (2012), which corresponds to the choice of $g_{3}=g_{3, \mathrm{cr}}$, we obtain the relations

$$
\frac{R_{m}}{R}=\frac{m(m+2)}{(m+1)^{2}}, \quad \frac{S_{m}}{S}=1-\frac{g_{3} R^{2}}{S} \frac{2}{(m+1)(m+2)}, \quad m \in \mathbb{N} .
$$

Since $C=2 \sqrt{R}+S$ and $2 g_{3, \mathrm{cr}} R^{3 / 2}=1$, see (6.7) of Bouttier and Guitter (2012), Equation (5.1) turns into

$$
\tilde{g}(m)\left(2+\frac{1}{(m+1)(m+2)}-\tilde{g}(m+1)\right)=\frac{m(m+2)}{(m+1)^{2}},
$$

or, with $\tilde{f}(m)=1-\tilde{g}(m)$,

$$
\tilde{f}(m)-\tilde{f}(m+1)+\tilde{f}(m) \tilde{f}(m+1)+\frac{\tilde{f}(m)-1}{(m+1)(m+2)}=\frac{1}{(m+1)^{2}} .
$$


Of course, the last display resembles very much Equation (3.3) for $f$, and in fact, $\tilde{f}(m)=1 /(m+1)$ is also a solution of (5.2). Rewriting (5.2) as

$$
\tilde{f}(m+1)=\frac{(m+1)^{2} \tilde{f}(m)-1}{(m+1)^{2}(1-\tilde{f}(m))}-\frac{1}{(m+1)(m+2)},
$$

we check with the same arguments as in the proof of Lemma 3.2 that $\tilde{f}(m)=$ $1 /(m+1)$ is the only solution of (5.2) with $\tilde{f}(m) \in(0,1)$ for $m \in \mathbb{N}, \tilde{f}(0)=1$ and $\lim _{m \rightarrow \infty} \tilde{f}(m)=0$. This shows $\mathbb{P}\left(\tilde{\Delta}_{0} \geq m\right)=1 /(m+1)$, as claimed. The law of $\tilde{\Delta}_{0}^{\prime}$ is now computed as in the proof of Proposition 3.1, using

$$
\mathbb{P}\left(\tilde{\Delta}_{0}<m\right)=\mathbb{P}\left(\tilde{\Delta}_{0}^{\prime}<m\right) \frac{R_{m}}{R}=\mathbb{P}\left(\tilde{\Delta}_{0}^{\prime}<m\right) \frac{m(m+2)}{(m+1)^{2}} .
$$

With the last proposition at hand, we obtain with the arguments given in the proof of Proposition 3.4 that

$$
\mathbb{P}\left(i \in \tilde{\mathcal{R}}_{+}\right)=\frac{1}{i+1}, \quad \mathbb{P}\left(i \in \tilde{\mathcal{R}}_{-}\right)=\frac{2}{i+2}, \quad i \in \mathbb{Z}_{+} .
$$

In particular, we again deduce that $\tilde{\gamma}_{\max }$ hits both parts of the boundary in the UIHPT infinitely many times. More precisely, comparing the last display with the analogous results obtained for $\mathcal{R}_{+}$and $\mathcal{R}_{-}$, we conclude that the intersection times of $\tilde{\gamma}_{\max }$ with the right part of the boundary have exactly the same distribution as the corresponding times of $\gamma_{\max }$ in the UIHPQ. On the contrary, the maximal geodesic visits the left part of the boundary slightly more often in the UIHPQ than in the UIHPT.

A symmetry argument similar to above shows that $\tilde{\mathcal{R}}_{+}^{\text {min }}$ has the same law as $\tilde{\mathcal{R}}_{-}$, and we have the inclusions $\tilde{\mathcal{R}}_{+} \subset \tilde{\mathcal{R}}_{+}^{\text {min }}$ and $\tilde{\mathcal{R}}_{-}^{\text {min }} \subset \tilde{\mathcal{R}}_{-}$. Using that $\tilde{\gamma}_{\text {max }}$ is proper and hits both parts of the boundary infinitely many times, we deduce from arguments very close to those in the proof of Corollary 3.6 that almost surely, all geodesic rays in the UIHPT are proper. Finally, adapting the arguments leading to Theorem 1.1 and Proposition 4.3, we arrive at the following theorem, whose details of proof we leave to the reader. We write $\tilde{Q}_{\infty}^{\infty}$ for the UIHPT constructed in terms of an uniform infinite mobile bridge $\left(\tilde{\mathrm{b}}_{\infty}, \tilde{T}_{\infty}\right)$.

Theorem 5.3. On a set of full probability, the following holds in the UIHPT $\tilde{Q}_{\infty}^{\infty}$ : Every geodesic ray hits the boundary of the UIHPT infinitely many times. Moreover, if $\gamma=\left(\gamma(i): i \in \mathbb{Z}_{+}\right)$is a geodesic ray emanating from the root vertex, we have the inclusions

$$
\tilde{\mathcal{R}}_{+} \cup \tilde{\mathcal{R}}_{-}^{\text {min }} \subseteq\left\{i \in \mathbb{Z}_{+}: \gamma(i) \in \partial \tilde{Q}_{\infty}^{\infty}\right\} \subseteq \tilde{\mathcal{R}}_{+}^{\min } \cup \tilde{\mathcal{R}}_{-} .
$$

The random sets $\tilde{\mathcal{R}}_{+}$and $\tilde{\mathcal{R}}_{-}^{\text {min }}$ (as well as $\tilde{\mathcal{R}}_{+}^{\text {min }}$ and $\tilde{\mathcal{R}}_{-}$) have the same law. The distance $\delta$ between two consecutive times in $\tilde{\mathcal{R}}_{+}$(or $\tilde{\mathcal{R}}_{-}^{\text {min }}$ ) exhibits the tail behavior $\mathbb{P}(\delta>m) \sim 1 / \ln m$ as $m \rightarrow \infty$, whereas the distance $\delta^{\prime}$ between two consecutive times in $\tilde{\mathcal{R}}_{+}^{\min }$ (or $\left.\tilde{\mathcal{R}}_{-}\right)$satisfies $\mathbb{P}\left(\delta^{\prime}>m\right) \sim 1 /(2 \ln m)$.

Concluding remarks. Angel constructed in Angel (2005) the uniform infinite triangulation with an infinite simple boundary, and we expect that Theorem 5.3 can be transferred to the model of Angel by a pruning procedure, as in the case of the UIHPQ ${ }^{(s)}$. Moreover, since the above construction of the UIHPT (or the UIHPQ) can be extended to general limits of critical or sub-critical Boltzmann maps, the 
same methods can in principle be applied to study the intersection of geodesic rays with the boundary for the full class of models obtained in this way.

However, as it should be clear from Remark 3.5, intersection properties of geodesics as studied in this paper are delicate, and our approach requires exact calculations (or at least non-asymptotic bounds). In the pure quadrangular and triangular cases at criticality, the expressions for $R_{m}$ and $S_{m}$ are particularly simple, so that we can compute the laws of $\Delta_{0}$ and $\tilde{\Delta}_{0}$ explicitly. See (5.11) of Bouttier and Guitter (2012) for the general form of $R_{m}$, which involves so-called Hankel determinants. For a more general treatment, Equation (5.1) is model-independent and may serve as a starting point for further investigations.

\section{Acknowledgements}

We would like to thank Nicolas Curien for asking us whether geodesic rays in the UIHPQ intersect the boundary infinitely often. Moreover, we thank Jérémie Bouttier for a helpful discussion, and the referee for pointing us to Chassaing and Durhuus (2006, Proposition 2.4).

\section{References}

O. Angel. Scaling of percolation on infinite planar maps, I. ArXiv Mathematics e-prints (2005). arXiv: 0501006.

O. Angel and N. Curien. Percolations on random maps I: Half-plane models. Ann. Inst. Henri Poincaré Probab. Stat. 51 (2), 405-431 (2015). MR3335009.

O. Angel and O. Schramm. Uniform infinite planar triangulations. Comm. Math. Phys. 241 (2-3), 191-213 (2003). MR2013797.

E. Baur, G. Miermont and G. Ray. Classification of scaling limits of uniform quadrangulations with a boundary. ArXiv Mathematics e-prints (2016). arXiv: 1608.01129.

J. Bouttier, P. Di Francesco and E. Guitter. Planar maps as labeled mobiles. Electron. J. Combin. 11 (1), Research Paper 69, 27 (2004). MR2097335.

J. Bouttier and E. Guitter. Planar maps and continued fractions. Comm. Math. Phys. 309 (3), 623-662 (2012). MR2885603.

A. Caraceni and N. Curien. Geometry of the uniform infinite half-planar quadrangulation. ArXiv Mathematics e-prints (2015). arXiv: 1508.00133.

P. Chassaing and B. Durhuus. Local limit of labeled trees and expected volume growth in a random quadrangulation. Ann. Probab. 34 (3), 879-917 (2006). MR2243873.

N. Curien, L. Ménard and G. Miermont. A view from infinity of the uniform infinite planar quadrangulation. ALEA Lat. Am. J. Probab. Math. Stat. 10 (1), 45-88 (2013). MR3083919.

N. Curien and G. Miermont. Uniform infinite planar quadrangulations with a boundary. Random Structures Algorithms 47 (1), 30-58 (2015). MR3366810.

W. Feller. An introduction to probability theory and its applications. Vol. I. Third edition. John Wiley \& Sons, Inc., New York-London-Sydney (1968). MR0228020.

P. Flajolet and R. Sedgewick. Analytic combinatorics. Cambridge University Press, Cambridge (2009). ISBN 978-0-521-89806-5. MR2483235. 
M. Krikun. Local structure of random quadrangulations. ArXiv Mathematics eprints (2005). arXiv: 05123049.

J.-F. Le Gall and G. Miermont. Scaling limits of random trees and planar maps. In Probability and statistical physics in two and more dimensions, volume 15 of Clay Math. Proc., pages 155-211. Amer. Math. Soc., Providence, RI (2012). MR3025391.

G. Miermont. An invariance principle for random planar maps. In Fourth Colloquium on Mathematics and Computer Science Algorithms, Trees, Combinatorics and Probabilities, Discrete Math. Theor. Comput. Sci. Proc., AG, pages 39-57. Assoc. Discrete Math. Theor. Comput. Sci., Nancy (2006). MR2509622.

G. Schaeffer. Conjugaison d'arbres et cartes combinatoires aléatoires. Ph.D. thesis, L'Université Bordeaux I (1998).

F. Spitzer. Principles of random walk. Springer-Verlag, New York-Heidelberg, second edition (1976). Graduate Texts in Mathematics, Vol. 34. MR0388547. 